\title{
A MEASUREMENT OF THE RATE OF TYPE Ia SUPERNOVAE IN GALAXY CLUSTERS FROM THE SDSS-II SUPERNOVA SURVEY
}

\author{
Benjamin Dilday ${ }^{1,2,3}$, Bruce Bassett ${ }^{4,5}$, Andrew Becker ${ }^{6}$, RAlF Bender $^{7,8}$, Francisco Castander $^{9}$, David Cinabro $^{10}$, \\ Joshua A. Frieman ${ }^{11,12}$, Lluís Galbany ${ }^{13}$, Peter Garnavich ${ }^{14}$, Ariel Goobar ${ }^{15,16}$, Ulrich Hopp $^{7,8}$, Yutaka Ihara ${ }^{17}$, \\ Saurabh W. Jha ${ }^{1}$, Richard Kessler ${ }^{3,11}$, Hubert Lampeitl ${ }^{18}$, John Marriner ${ }^{12}$, Ramon Miquel ${ }^{13,19}$, Mercedes Mollá ${ }^{20}$, \\ Robert C. Nichol ${ }^{18}$, Jakob Nordin ${ }^{16}$, AdAm G. Riess ${ }^{21,22}$, Masao SaKo ${ }^{23}$, Donald P. Schneider ${ }^{24}$, MATHew SMith $^{4,18}$, \\ Jesper Sollerman ${ }^{15,25}$, J. Craig WheEler ${ }^{26}$, Linda Östman ${ }^{16}$, Dmitry Bizyaev ${ }^{27}$, Howard Brewington ${ }^{27}$, \\ Elena Malanushenko ${ }^{27}$, Viktor Malanushenko ${ }^{27}$, Dan Oravetz ${ }^{27}$, Kaike Pan $^{27}$, Audrey Simmons $^{27}$, \\ AND STEPhanie SNEDDEN ${ }^{27}$ \\ ${ }^{1}$ Department of Physics and Astronomy, Rutgers, The State University of New Jersey, 136 Frelinghuysen Road, Piscataway, NJ 08854, USA; \\ bdilday@physics.rutgers.edu \\ ${ }^{2}$ Department of Physics, University of Chicago, Chicago, IL 60637, USA \\ ${ }^{3}$ Kavli Institute for Cosmological Physics, The University of Chicago, 5640 South Ellis Avenue Chicago, IL 60637, USA \\ ${ }^{4}$ Department of Mathematics and Applied Mathematics, University of Cape Town, Rondebosch 7701, South Africa \\ ${ }^{5}$ South African Astronomical Observatory, P.O. Box 9, Observatory 7935, South Africa \\ ${ }^{6}$ Department of Astronomy, University of Washington, Box 351580, Seattle, WA 98195, USA \\ ${ }^{7}$ Max Planck Institute for Extraterrestrial Physics, D-85748, Garching, Munich, Germany \\ ${ }^{8}$ Universitaets-Sternwarte Munich, 1 Scheinerstr, Munich, D-81679, Germany \\ ${ }^{9}$ Institut de Ciències de l'Espai (IEEC-CSIC), Barcelona, Spain \\ ${ }^{10}$ Department of Physics and Astronomy, Wayne State University, Detroit, MI 48202, USA \\ ${ }^{11}$ Department of Astronomy and Astrophysics, The University of Chicago, 5640 South Ellis Avenue, Chicago, IL 60637, USA \\ ${ }^{12}$ Center for Astrophysics, Fermi National Accelerator Laboratory, P.O. Box 500, Batavia IL 60510, USA \\ ${ }^{13}$ Institut de Física d'Altes Energies, Barcelona, Spain \\ ${ }^{14}$ University of Notre Dame, 225 Nieuwland Science, Notre Dame, IN 46556-5670, USA \\ 15 The Oskar Klein Centre, Department of Astronomy, Albanova, Stockholm University, SE-106 91 Stockholm, Sweden \\ ${ }^{16}$ Department of Physics, Stockholm University, Albanova University Center, S-106 91 Stockholm, Sweden \\ ${ }^{17}$ Institute of Astronomy, Graduate School of Science, University of Tokyo 2-21-1, Osawa, Mitaka, Tokyo 181-0015, Japan \\ ${ }^{18}$ Institute of Cosmology and Gravitation, Mercantile House, Hampshire Terrace, University of Portsmouth, Portsmouth PO1 2EG, UK \\ ${ }^{19}$ Institució Catalana de Recerca i Estudis Avançats, Barcelona, Spain \\ ${ }^{20}$ Centro de Investigaciones Energéticas, Medioambientales y Tecnol ógicas, Madrid, Spain \\ ${ }^{21}$ Space Telescope Science Institute, 3700 San Martin Drive, Baltimore, MD 21218, USA \\ ${ }^{22}$ Department of Physics and Astronomy, Johns Hopkins University, 3400 North Charles Street, Baltimore, MD 21218, USA \\ ${ }^{23}$ Department of Physics and Astronomy, University of Pennsylvania, 209 South 33rd Street, Philadelphia, PA 19104, USA \\ ${ }^{24}$ Department of Astronomy and Astrophysics, 525 Davey Laboratory, Pennsylvania State University, University Park, PA, USA \\ ${ }^{25}$ Dark Cosmology Centre, Niels Bohr Institute, University of Copenhagen, Denmark \\ ${ }^{26}$ Department of Astronomy, University of Texas, Austin, TX 78712, USA \\ ${ }^{27}$ Apache Point Observatory, P.O. Box 59, Sunspot, NM 88349, USA \\ Received 2009 July 20; accepted 2010 April 3; published 2010 May 6
}

\section{ABSTRACT}

We present measurements of the Type Ia supernova (SN) rate in galaxy clusters based on data from the Sloan Digital Sky Survey-II (SDSS-II) Supernova Survey. The cluster SN Ia rate is determined from 9 SN events in a set of $71 \mathrm{C} 4$ clusters at $z \leqslant 0.17$ and $27 \mathrm{SN}$ events in $492 \mathrm{maxBCG}$ clusters at $0.1 \leqslant z \leqslant 0.3$. We find values for the cluster SN Ia rate of $\left(0.37_{-0.12-0.01}^{+0.17+0.01}\right) \mathrm{SNu} r h^{2}$ and $\left(0.55_{-0.11-0.01}^{+0.13+0.02}\right) \mathrm{SNu} h^{2}\left(\mathrm{SNu} x=10^{-12} L_{x \odot}^{-1} \mathrm{yr}^{-1}\right)$ in C4 and maxBCG clusters, respectively, where the quoted errors are statistical and systematic, respectively. The SN rate for early-type galaxies is found to be $\left(0.31_{-0.12-0.01}^{+0.18+0.01}\right) \mathrm{SNu} r h^{2}$ and $\left(0.49_{-0.11-0.01}^{+0.15+0.02}\right) \mathrm{SNu} r h^{2}$ in $\mathrm{C} 4$ and maxBCG clusters, respectively. The $\mathrm{SN}$ rate for the brightest cluster galaxies (BCG) is found to be $\left(2.04_{-1.11-0.04}^{+1.99+0.07}\right) \mathrm{SNu} r h^{2}$ and $\left(0.36_{-0.30-0.01}^{+0.84+0.01}\right) \mathrm{SNu} r h^{2}$ in $\mathrm{C} 4$ and maxBCG clusters, respectively. The ratio of the SN Ia rate in cluster early-type galaxies to that of the SN Ia rate in field early-type galaxies is $1.94_{-0.91-0.015}^{+1.31+0.043}$ and $3.02_{-1.03-0.048}^{+1.31+0.062}$, for $\mathrm{C} 4$ and maxBCG clusters, respectively. The SN rate in galaxy clusters as a function of redshift, which probes the late time SN Ia delay distribution, shows only weak dependence on redshift. Combining our current measurements with previous measurements, we fit the cluster SN Ia rate data to a linear function of redshift, and find $r_{L}=\left[\left(0.49_{-0.14}^{+0.15}\right)+\left(0.91_{-0.81}^{+0.85}\right) \times z\right] \mathrm{SNu} B$ $h^{2}$. A comparison of the radial distribution of SNe in cluster to field early-type galaxies shows possible evidence for an enhancement of the SN rate in the cores of cluster early-type galaxies. With an observation of at most three hostless, intra-cluster SNe Ia, we estimate the fraction of cluster SNe that are hostless to be $\left(9.4_{-5.1}^{+8.3}\right) \%$.

Key words: supernovae: general

\section{INTRODUCTION}

The rate of Type Ia supernovae ( $\mathrm{SNe}$ ) in galaxy clusters is an important field of study for a number of reasons. As discussed in, e.g., Greggio (2005), supernova (SN) rate measurements are an observational probe of the progenitor systems, with the connection to progenitor models being made through inference of the distribution of delay times (DDTs) with respect to star formation. As galaxy clusters are generally composed of a high fraction of early-type galaxies that have old stellar populations, measurements of the SN Ia rate in clusters can in principle simplify the inference of the SN DDT. SNe in galaxy clusters are also a candidate source for metal enrichment of the intra-cluster medium (ICM). In particular, improved measurement of the 
Table 1

Cluster Rate Measurements

\begin{tabular}{|c|c|c|c|c|c|}
\hline Reference & $\begin{array}{l}\text { Redshift } \\
\text { Range }\end{array}$ & $\begin{array}{c}\text { Mean } \\
\text { Redshift }\end{array}$ & $\begin{array}{c}\text { Lookback Time } \\
\text { (Gyr) }\end{array}$ & $N_{\mathrm{SNe}}$ & $\begin{array}{l}\text { SN Ia Rate }^{\mathrm{a}} \\
\left(\mathrm{SNuB} h^{2}\right)\end{array}$ \\
\hline This work (C4) & $0.03-0.17$ & 0.084 & 1.11 & 9 & $0.46_{-0.15-0.01}^{+0.21+0.01}$ \\
\hline This work (maxBCG) & $0.10-0.30$ & 0.225 & 2.69 & 25 & $0.68_{-0.14-0.02}^{+0.17+0.02}$ \\
\hline Mannucci et al. (2008) & $0-0.04$ & 0.020 & 0.28 & 12.5 & $0.57_{-0.16}^{+0.22}$ \\
\hline Sharon et al. (2007a) & $0.06-0.19$ & 0.150 & 1.89 & 6 & $0.73_{-0.29}^{+0.45}$ \\
\hline Gal-Yam et al. (2002) & $\approx 0.25$ & 0.250 & 2.94 & 1 & $0.80_{-0.65}^{+1.84}$ \\
\hline Graham et al. (2008) & $\approx 0.45$ & 0.450 & 4.67 & 3 & $0.63_{-0.33}^{+1.04}$ \\
\hline Gal-Yam et al. (2002) & $\approx 0.9$ & 0.900 & 7.30 & 2 & $1.63_{-1.06}^{+2.16}$ \\
\hline
\end{tabular}

Note. ${ }^{\text {a }}$ For this work, the quoted errors are statistical and systematic, respectively. For previous measurements, the total error is quoted; see the corresponding references.

rate of intra-cluster SNe would be significant for constraining the relative importance of sources that may contribute to the cluster ICM enrichment (e.g., intra-cluster stars versus galaxy outflow).

The existing measurements of the cluster SN rate are few and are generally based on low-number statistics. Estimates of the SN cluster rate were first presented by Crane et al. (1977) and Barbon (1978), who considered $\approx 5 \mathrm{SNe}$ discovered in the Coma cluster. The cluster SN rate was measured by Gal-Yam et al. (2002) in clusters at $z \approx 0.25$ and $z \approx 0.9$. The Gal-Yam et al. (2002) results are based on a search for $\mathrm{SNe}$ in archival images of the Hubble Space Telescope (HST) and utilize one and two $\mathrm{SNe}$, respectively. Subsequent to the HST SN search, a dedicated search for SNe in 161 Abell clusters was undertaken by The Wise Observatory Optical Transient Search (WOOTS; Gal-Yam et al. 2008). A total of six SNe Ia discovered by the WOOTS were used to determine the cluster SN Ia rate at $z \approx 0.15$ by Sharon et al. (2007a). A sample of 2-3 SNe Ia from the Supernova Legacy Survey (SNLS) have been used to determine the cluster SN Ia rate at $z \approx 0.45$ by Graham et al. (2008). Finally, the sample of $\mathrm{SNe}$ in the local $(z \lesssim 0.04)$ universe presented by Cappellaro et al. (1999) have been reanalyzed by Mannucci et al. (2008) to determine the cluster SN Ia rate with a sample of 12.5 SN Ia (a fractional SN reflects uncertainty in typing; see Cappellaro et al. 1999). Additionally, Mannucci et al. (2008) have placed the first constraints on the core-collapse (CC) SN rate in galaxy clusters based on a sample of 7.5 CC SNe. A summary of the SN Ia rate results from the above cluster SN studies is given in Table 1. A SN search in 15 massive, high-redshift $(0.5<z<0.9)$, $\mathrm{X}$-ray-selected clusters has been carried out on the HST, as described in Sharon et al. (2007b), and a measurement of the $\mathrm{SN}$ Ia rate based on 6-14 $\mathrm{SNe}$ discovered by the program is forthcoming (K. Sharon et al. 2010, in preparation). A dedicated $\mathrm{SN}$ search, targeting $\approx 60 \mathrm{X}$-ray-selected clusters in the redshift range $0.1<z<0.2$, is also being carried out on the Bok 2. 3 $m$ telescope on Kitt Peak (Sand et al. 2008).

As can be seen in Table 1, the knowledge of the cluster SN Ia rate comprises five measurements, based on a total of $\approx 25 \mathrm{SNe}$. In this paper, we describe new measurements of the cluster $\mathrm{SN}$ Ia rate based on data from the Sloan Digital Sky Survey-II (SDSS-II) Supernova Survey (Frieman et al. 2008). The measurements are based on $35 \mathrm{SNe}$ in the redshift range $0.03<z<0.30$, and therefore represent a significant statistical contribution to cluster SN Ia studies. In Section 2, we briefly describe the observations and SN search strategy of the SDSS-II Supernova Survey. In
Section 3, we describe the galaxy cluster catalogs employed in this SN rate analysis. In Section 4, we describe selection of the cluster SN sample from the SDSS-II Supernova Survey data. In Section 5, we describe necessary corrections to our SN Ia rate measurements. In Section 7, we present results on the cluster SN Ia rate, as well as limits on the cluster CC SN rate, and studies of the distribution of $\mathrm{SNe}$ with respect to their host galaxies. We summarize in Section 8. Whenever necessary we assume a flat $\Lambda$ CDM cosmology with $\Omega_{\mathrm{m}}=1-\Omega_{\Lambda}=0.3$, and Hubble constant $H_{0}=70 \mathrm{~km} \mathrm{~s}^{-1} \mathrm{Mpc}^{-1}$.

\section{SDSS-II SUPERNOVA SURVEY OBSERVATIONS}

Here we briefly describe aspects of the SDSS-II Supernova Survey most relevant to the present SN rate analysis. Much of the material presented in this section is also relevant to the $\mathrm{SN}$ rate studies described in Dilday et al. (2010), and is discussed therein; we repeat the discussion here for the convenience of the reader. The survey is described in more detail in Frieman et al. (2008) and the SN detection algorithms are described in Sako et al. (2008). Additional details of the survey observations and the use of in situ artificial SNe for determining SN detection efficiencies are discussed in Dilday et al. (2008). A technical summary of the SDSS is given by York et al. (2000). Details of the survey calibration can be found in Hogg et al. (2001), Smith et al. (2002), and Tucker et al. (2006), the data processing and quality assessment is described by Ivezić et al. (2004), and the photometric pipeline is described by Lupton et al. (1999).

The SDSS-II Supernova Survey was carried out during the Fall (September-November) of 2005-2007, using the $2.5 \mathrm{~m}$ telescope (Gunn et al. 2006) at Apache Point Observatory (APO). Observations were obtained in the SDSS ugriz filters (Fukugita et al. 1996) with a wide-field CCD camera (Gunn et al. 1998), operating in time-delay-and-integrate (TDI, or drift scan) mode. The region of the sky covered by the SDSS-II Supernova Survey (designated stripe 82; see Stoughton et al. 2002) was bounded by $-60^{\circ}<\alpha_{J 2000}<60^{\circ}$, and $-1.258<$ $\delta_{J 2000}<1$.258. On average any given part of this $\approx 300$ square degree area was imaged once every 4 days during the survey operations.

Difference images were produced in the SDSS gri filter bands by subtracting template images, constructed from previous observation of the survey region, from the survey images, using an implementation of the methods described by Alard \& Lupton (1998). The difference images were searched for positive fluctuations using the DoPHOT photometry and object detection 
package (Schechter et al. 1993); typical limiting magnitudes (10 $\sigma$ above background) for the SDSS-II Supernova Survey were $g \sim 21.8, r \sim 21.5$, and $i \sim 21.2$. A combination of software cuts and human visual inspection was then used to identify promising SN candidates from the full set of transient detections. As a key component of prioritizing SN candidates for spectroscopic observation, the light curves for $\mathrm{SN}$ candidates were fit to models of Type Ia, Type Ib/c, and Type II SNe. This procedure is referred to as photometric-typing, and is described in detail by Sako et al. (2008).

Spectroscopic observations, for both SN type and redshift determination, were provided by a number of different telescopes. The spectra for the SNe utilized in the present $\mathrm{SN}$ rate analysis were provided by the Hobby-Eberly $9.2 \mathrm{~m}$ at McDonald Observatory, the Astrophysical Research Consortium $3.5 \mathrm{~m}$ at Apache Point Observatory, the William-Herschel $4.2 \mathrm{~m}$, the Hiltner $2.4 \mathrm{~m}$ at the MDM Observatory, the Subaru $8.2 \mathrm{~m}$ on Mauna Kea, the $2.6 \mathrm{~m}$ Nordic Optical Telescope and the $3.6 \mathrm{~m}$ Italian Telescopio Nazionale Galileo at La Palma, the Mayall $4 \mathrm{~m}$ telescope at Kitt Peak National Observatory, and the $3.5 \mathrm{~m}$ ESO New Technology Telescope (NTT) at La Silla. Details of the SDSS-II Supernova Survey spectroscopic data reductions are given by Zheng et al. (2008). Comparison to high-quality SDSS galaxy spectra show that SN spectroscopic redshifts are accurate to $\approx 0.0005$ when galaxy emission features are used and $\approx 0.005$ when $\mathrm{SN}$ features are used. In either case the error on the spectroscopic $\mathrm{SN}$ redshifts is negligible for the $\mathrm{SN}$ rate studies considered here.

While the difference imaging pipeline used during the SN search provides initial photometric measurements, subsequent to the search more precise SN photometry is provided using a scene modeling photometry (SMP) technique developed by Holtzman et al. (2008). The final analysis of SN light curves and the selection cuts used to define the SN rate sample discussed in this paper are based on SMP.

\section{GALAXY CLUSTER CATALOGS AND CLUSTER LUMINOSITY}

In studying the Type Ia SN rate in galaxy clusters we will work with two primary cluster catalogs; the $\mathrm{C} 4$ cluster catalog and the maxBCG cluster catalog. The $\mathrm{C} 4$ cluster finding algorithm and catalog are discussed in detail by Miller et al. (2005). The maxBCG catalog is presented by Koester et al. (2007a), and the cluster finding algorithm is described by Koester et al. (2007b). We briefly describe and summarize the content of these two cluster catalogs below. The redshift distributions for the clusters in these two catalogs that are in the SDSS-II Supernova Survey region are shown in Figure 1.

\subsection{The C4 Cluster Catalog Description}

The C4 cluster catalog is based on the main spectroscopic sample of the SDSS and contains clusters in the redshift range $0.03<z<0.17$. The $\mathrm{C} 4$ cluster catalog provides, among other quantities, cluster coordinates, the luminosity of each cluster in the SDSS $r$ band, and the number of galaxies identified as members of each cluster, $N_{\text {gals }}$. The main spectroscopic sample of galaxies from SDSS is designed to be complete to a limiting magnitude of $r \approx 17.8$ (Strauss et al. 2002). For typical cluster galaxy luminosities, this implies that the identification of member galaxies, with $L>0.4 L_{\star}$, is complete for clusters at $z<0.11$. For clusters above this redshift limit a correction has to be made to the total cluster luminosity. The $\mathrm{C} 4$

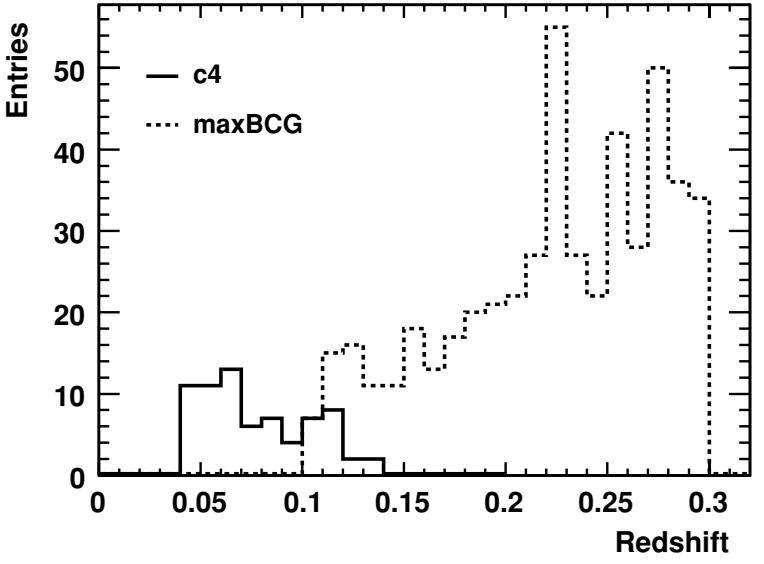

Figure 1. Redshift distributions for the C4 $(z<0.17)$ and maxBCG $(0.1<z<$ $0.3)$ clusters in the SDSS-II SN Survey region. There are 71 clusters from the C4 catalog and 492 clusters from the maxBCG catalog.

cluster identification algorithm works by searching for groups of objects that are tightly clustered in a seven-dimensional feature space that includes spatial position, redshift, and observed colors. Note that there is no requirement that the colors for the galaxies be consistent with the colors of early-type galaxies, only that they be consistent with one another. The SDSS main galaxy sample cannot be $100 \%$ complete, as the SDSS fiberspectrograph imposes a minimum angular separation of $55^{\prime \prime}$ $\left(\approx 100 \mathrm{kpc}^{-1}\right.$ at $\left.z=0.15\right)$ for objects targeted for the SDSS main spectroscopic galaxy sample (Stoughton et al. 2002). A correction is applied to the $\mathrm{C} 4$ cluster luminosities to account for this by including galaxies as members of the cluster when they satisfy the magnitude requirement $(r<17.8)$ and have similar colors to the spectroscopically determined cluster members. The published C4 catalog is based on the 2nd data release of the SDSS (Abazajian et al. 2004) and contains 748 clusters. The catalog used in this work is an extended version of the $\mathrm{C} 4$ catalog that contains 1713 clusters and is based on the 5th data release of the SDSS (Adelman-McCarthy et al. 2007; B. Nichol 2007, private communication). The subset of clusters that we will use in measuring the cluster $\mathrm{SN}$ rate are those that lie within stripe 82 , and that have $-40^{\circ}<\alpha_{\mathrm{J} 2000}<50^{\circ}$. There are $71 \mathrm{C} 4$ clusters in this subset.

\subsection{The maxBCG Cluster Catalog Description}

The maxBCG catalog is based on SDSS photometric measurements, and the cluster identification algorithm relies on the tight relationship between color and redshift for luminous red galaxies, which make up the majority of galaxy cluster composition. The maxBCG algorithm assigns a photometric redshift to each identified cluster that is derived by comparing the cluster member galaxy colors to the expected colors for early-type galaxies, as a function of redshift. Comparison of the photometric redshifts for the maxBCG clusters to the spectroscopically measured redshift of the brightest cluster galaxy (BCG), when available, shows that the residuals for redshift (photometric-spectroscopic) are well described by a Gaussian distribution with a mean of 0 and a standard deviation of $\sigma \approx 0.015$. The observed $(g-r)$ and $(r-i)$ colors for maxBCG member galaxies, as a function of redshift, are shown in Figure 2. Linear functions were fit to the $(r-i)$ and $(g-r)$ colors as a function of redshift, $z$, and these will be used below for applying $k$-corrections to the observations. For reference, the 


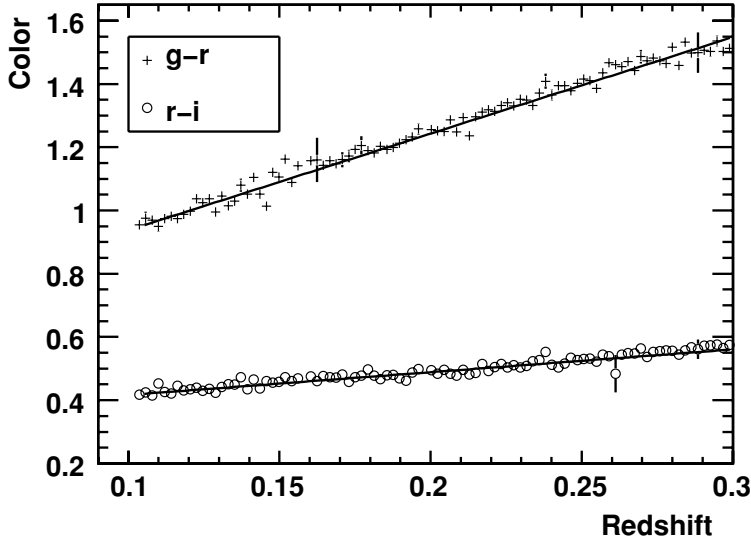

Figure 2. Observed colors of maxBCG galaxies vs. redshift. The points represent the mean at the corresponding redshift, and the error bars represent the error on the mean. The solid lines show the best-fitting linear function.

functions derived, valid for $0.1 \leqslant z \leqslant 0.3$, are,

$$
\begin{aligned}
& (r-i)=0.345+0.720 z \\
& (g-r)=0.632+3.054 z .
\end{aligned}
$$

The maxBCG catalog is restricted to the redshift interval $0.1<z<0.3$, and is thus highly complementary to the $\mathrm{C} 4$ catalog. The lower limit for the maxBCG cluster catalog is imposed because the fractional photometric redshift errors for redshifts less than $z=0.1$ are significant and have a large systematic effect on derived cluster properties. Furthermore, cluster catalogs based on spectroscopic redshifts for $z<0.1$ are already available. The upper limit is imposed because, at $z \approx 0.3$, the "4000 $\AA$ break" that is responsible for the uniformity in early-type galaxy colors moves into the region between the SDSS observer frame $g$ - and $r$-band filters. Thus, the accuracy and precision for galaxy photometric redshifts is severely diminished (Koester et al. 2007a). A lower limit for the luminosity of cluster members is imposed so that the definition of the composition of the clusters is consistent across the redshift range. The limit corresponds to an absolute magnitude of ${ }^{(0.25)} i \approx-20.25$, (the ${ }^{(0.25)} i$ notation is explained in Section 3.3) and is such that the catalog is volume limited over the entire redshift range $0.1<z<0.3$. Measures of the physical extent, $r_{200}$, and of the richness, $N_{200}$ and $L_{200}$, are provided for each cluster. $r_{200}$ is defined as the radius such that the mean density of early-type galaxies contained within is 200 times greater than the mean density of such galaxies (Koester et al. 2007a); $N_{200}\left(L_{200}\right)$ is defined as the number (total luminosity) of early-type galaxies contained within $r_{200}$. The public maxBCG cluster catalog contains 13,823 clusters with $N_{200} \geqslant 10$. The subset of maxBCG clusters that we will use in measuring the cluster $\mathrm{SN}$ rate are those that lie within stripe 82 , and that have $-50^{\circ}<\alpha_{\mathrm{J} 2000}<60^{\circ}$. There are 492 maxBCG clusters in this subset. Additionally, this work makes use of a catalog of the maxBCG member galaxies (B. Koester 2007 , private communication).

In addition to the cluster richness and luminosity estimates that are provided with the maxBCG catalog, the maxBCG clusters have been extensively studied by, e.g., Sheldon et al. (2009a, 2009b) and Johnston et al. (2007). Of particular relevance to the study of the SN rate in galaxy clusters are the luminosity functions (LFs) of maxBCG clusters presented by Hansen et al. (2009), and we will make extensive use of these below.

\subsection{Luminosity Content of C4 and maxBCG Cluster Catalogs}

In Section 7, we will present the $\mathrm{SN}$ rate in galaxy clusters per unit luminosity. In this section, we present a comprehensive discussion of the luminosity content for the $\mathrm{C} 4$ and maxBCG cluster catalogs. In what follows we use the notation ${ }^{(z)} m$ to denote the observer frame magnitude, $m$, for an object that has been $k$-corrected to a redshift $z$. All luminosities presented here are for galaxies with $L>0.4 L_{*}\left(L_{*}\right.$ is a characteristic luminosity for cluster members; see Equation (3)). Correcting for the faint end of the luminosity distribution is discussed below.

The C4 catalog provides total cluster luminosities in SDSS $r$ band. The total uncorrected $r$-band luminosity for the galaxies identified as cluster members, for clusters considered in this study, is $2.02 \times 10^{13} L_{\odot} h^{-2}$, where $L_{\odot}$ is the luminosity of the Sun, and $h$ is the value of the Hubble constant in units of $100 \mathrm{~km} \mathrm{~s}^{-1} \mathrm{Mpc}^{-1}$. As discussed in Section 3.1, these luminosities have to be corrected to account for incompleteness of the spectroscopic sample due to fiber collisions. The total $r$-band luminosity after making this correction is also provided by the $\mathrm{C} 4$ catalog and has the value $4.08 \times 10^{13} L_{\odot} h^{-2}$. As mentioned above, the luminosities for clusters at $z>0.11$ also have to be corrected to account for the fact that some cluster galaxies with $L>0.4 L_{\star}$ will have the observed magnitudes fainter than the completeness limit of the main SDSS galaxy sample $(r \approx 17.8)$. Of the $71 \mathrm{C} 4$ clusters we are considering, 12 are at $z>0.11$. Applying a correction to the luminosities of these 12 clusters, assuming the cluster LFs of Hansen et al. (2009), results in a total $r$-band luminosity for C4 clusters in this study of $4.12 \times 10^{13} L_{\odot} h^{-2}$.

The maxBCG catalog includes the summed luminosities for member galaxies in ${ }^{(0.25)} i$ and ${ }^{(0.25)} r$. However, the maxBCG cluster LFs presented by Hansen et al. (2009) represent a more complete study of the luminosity content of maxBCG clusters, and we will use these as the definitive measure of the total maxBCG cluster luminosities. In particular, a background subtraction has been performed that reduces inaccuracies in the cluster luminosities due to interloping foreground and background galaxies that may be counted as maxBCG cluster members. In Hansen et al. (2009), LFs are presented for maxBCG satellites, as a function of the richness measure, $N_{200}$. The LFs account for both red and blue cluster galaxies, but do not include the contribution to the luminosity from the BCG. The LFs are assumed to take the form of a Schechter function,

$$
\phi(L) d L=\phi^{*}\left(\frac{L}{L_{*}}\right)^{\alpha} e^{-L / L_{*}} \frac{d L}{L_{*}},
$$

where $L_{*}$ is a characteristic luminosity for cluster members, and $\phi^{*}$ is a normalization constant with units of inverse volume. The three parameters of the LFs, $\phi^{*}, L_{*}, \alpha$, are each expressed as functions of $N_{200}$, with the general functional form of $A \times N_{200}^{\beta}$. The values of $A$ (normalization) and $\beta$ (exponent) for these three LF parameters are given in Table 2 . To use the LFs to compute the total luminosity we exploit the identity

$$
\Sigma L=\left\langle L\left(N_{200}\right)\right\rangle N_{200},
$$

where $\Sigma L$ denotes the summed luminosity for the cluster, and the average luminosity, $\langle L\rangle$, is given by,

$$
\left\langle L\left(N_{200}\right)\right\rangle=\frac{\int_{0.4 L_{*}}^{\infty} d L L \phi(L)}{\int_{0.4 L_{*}}^{\infty} d L \phi(L)} .
$$


Table 2

maxBCG Luminosity Function Parameters

\begin{tabular}{lcr}
\hline \hline LF Parameter & $\begin{array}{c}\text { Normalization } \\
(\mathrm{A})\end{array}$ & $\begin{array}{c}\text { Exponent } \\
(\beta)\end{array}$ \\
\hline$\phi^{*}$ & $8.0 \mathrm{Mpc}^{-3} h^{3}$ & -0.20 \\
$L_{*}$ & $0.8 \times 10^{10} L_{\odot} h^{-2}$ & 0.15 \\
$\alpha$ & -0.28 & 0.25 \\
\hline
\end{tabular}

Note. Parameters refer to Schechter functions derived by Hansen et al. (2009).

Equation (4) gives the total ${ }^{(0.25)} i$ luminosity in a cluster, as a function of $N_{200}$. The total luminosity in clusters in the SN survey region is then the sum over $N_{200}$ of $\left\langle L\left(N_{200}\right)\right\rangle N_{\mathrm{SN}}\left(N_{200}\right)$, where $N_{\mathrm{SN}}\left(N_{200}\right)$ is the number of clusters in the survey region for which the number of member galaxies is $N_{200}$. Using this formalism we find that the total luminosity of maxBCG clusters in the survey region is $\Sigma L_{i}^{0.25}=1.096 \times 10^{14} L_{\odot} h^{-2}$.

\subsubsection{Correcting Cluster Luminosities for Faint Galaxies}

The luminosities quoted above for the $\mathrm{C} 4$ and maxBCG cluster catalogs include only galaxies with $L>0.4 L_{*}$, which is a conventional way of characterizing cluster luminosities. In measuring the cluster $\mathrm{SN}$ rate we do not wish to exclude $\mathrm{SNe}$ occurring in faint galaxies, and so it is necessary to estimate the contribution to the total cluster luminosities from galaxies with $L<0.4 L_{*}$. The total cluster luminosity can be estimated as $L=\kappa L^{+}$, where $L^{+}$denotes the luminosity for galaxies with $L>0.4 L_{*}$ and the correction factor, $\kappa$, is given by

$$
\kappa=\frac{\int_{0}^{\infty} d L L \phi(L)}{\int_{0.4 L_{*}}^{\infty} d L L \phi(L)}
$$

For a typical maxBCG cluster with $N_{200}=20$ the power-law exponent of the LF is $\alpha=-0.59$ (Table 2). For a cluster with $N_{200}=35$ the value for the exponent is $\alpha=-0.68$. The corresponding correction factors are $\kappa=1.21$ and $\kappa=1.25$. We will assume that the faint end of the LF is a characteristic property of galaxy clusters and that the power-law behavior of the LFs for maxBCG clusters is appropriate for the $\mathrm{C} 4$ clusters also.

\subsection{2. $k$-corrections}

In order to minimize uncertainties due to $k$-corrections, the maxBCG magnitudes, and the corresponding luminosities, have been $k$-corrected to the median redshift of the clusters, $z=0.25$. For comparison of the $\mathrm{SN}$ rate results based on the maxBCG clusters with previous cluster SN rate measurements, it will be necessary to $k$-correct the luminosities into more standard filters. In order to determine the appropriate $k$-corrections we selected a set of galaxies from the SDSS galaxy catalog that satisfies the color versus redshift relations for maxBCG members discussed above. The corresponding set of galaxies contains $\approx 675,000$ members. We then matched these galaxies with their corresponding records in the photoz database provided by the SDSS catalog archive server (CAS), which provides $k$-corrections based on the work of Blanton et al. (2003a). We thereby determine an average $k$-correction as a function of redshift, appropriate to the early-type galaxies that make up the bulk of the maxBCG catalog. The $k$-corrections so derived, in SDSS $r$ and $i$ bands, are shown in Figure 3. For reference, the

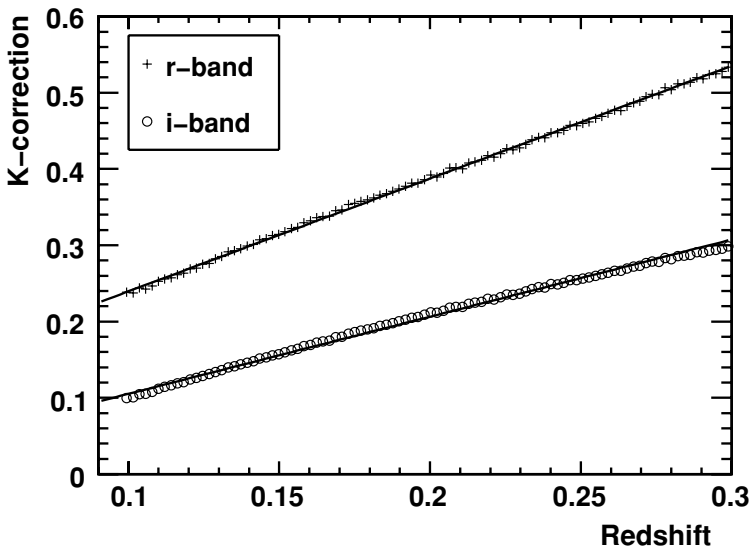

Figure 3. $k$-corrections vs. redshift for early-type galaxies. The points represent the mean at the corresponding redshift, and the error bars represent the error on the mean. The solid lines show the best-fitting linear function.

functions employed for $k$-corrections as a function of redshift, $z$, valid for $0.1 \leqslant z \leqslant 0.3$, are

$$
\begin{aligned}
& K_{r r}(z)=9.17 \times 10^{-2}+1.48 z, \\
& K_{i i}(z)=3.95 \times 10^{-3}+1.01 z .
\end{aligned}
$$

The functions above were derived by binning the $k$-corrections in redshift and taking the error on the mean (rms divided by square root of the number of entries) as the uncertainty. With this formalism the error on the fitted parameters is $\lesssim 1 \%$ and is thus negligible in comparison to the statistical error on our $\mathrm{SN}$ Ia rate measurements.

To transform the maxBCG cluster luminosities from ${ }^{(0.25)} i$ to $r$, we note the following identity:

$$
\begin{aligned}
m_{r} & =m_{r}^{0.25}-K_{r r}(z=0.25) \\
& =m_{i}^{0.25}+(r-i)(z=0.25)-K_{r r}(z=0.25) .
\end{aligned}
$$

We $k$-correct the maxBCG cluster luminosities from ${ }^{(0.25)} i$ to $r$ because at a redshift $z=0.25$ rest-frame $r$ maps closely to observer-frame $i$, and because the analysis of Hansen et al. (2009) that we take as the definitive measurement of the maxBCG cluster luminosities is performed for ${ }^{(0.25)} i$ and not ${ }^{(0.25)} r$. Using the expressions given above for $(r-i)$ and $K_{r r}$, evaluated at $z=0.25$, we derive the transformation $m_{r}={ }^{(0.25)} m_{i}+0.063$. For comparison to other cluster SN rate measurements, it is necessary to express the cluster luminosities in units of $L_{\odot}^{r}$. The conversion to solar luminosities is given by

$$
\left(\frac{L}{L_{\odot}}\right)_{r}=\left(\frac{L}{L_{\odot}}\right)_{0.25_{i}} \times 10^{-0.4\left(\left(m_{r}-m_{0.25_{i}}\right)-\left(M_{\odot}^{r}-M_{\odot}^{0.25_{i}}\right)\right)},
$$

where $M_{\odot}$ is the absolute magnitude of the Sun. To compute the absolute magnitude of the Sun in an arbitrary filter, we use a solar spectrum obtained from the CALSPEC ${ }^{28}$ database, hosted by the Space Telescope Science Institute, and compute synthetic magnitudes using a custom piece of software written for this task. We thereby derive values for the absolute magnitude of the Sun of $M_{\odot}^{r}=4.64$ (in agreement with the value published

\footnotetext{
28 http://www.stsci.edu/hst/observatory/cdbs/calspec.html
} 
by Blanton \& Roweis 2007), and $M_{\odot}^{0.25} i=4.67$. With these values, Equation $(11)$ becomes $\left(L / L_{\odot}\right)_{r}=0.918\left(L / L_{\odot}\right)_{0.25}$, giving the $r$-band luminosity in maxBCG clusters, for galaxies with $L>0.4 L_{*}$, as $\Sigma L_{r}=1.006 \times 10^{14} L_{\odot}$.

\subsection{Uncertainty on the Cluster Luminosities}

Any uncertainty on the luminosity of the clusters described above will propagate to an uncertainty on the derived cluster SN Ia rates. Sources of uncertainty on the cluster luminosities might include, e.g., uncertainties in the background subtraction used in defining the maxBCG cluster luminosities, or uncertainty in the correction for fiber collisions in defining the $\mathrm{C} 4$ clusters. The cluster catalogs described above do not include uncertainties on the cluster luminosities, and it is beyond the scope of this analysis to determine these uncertainties. However, as an estimate of the order of magnitude of the cluster luminosity uncertainties, we note that Menanteau et al. (2010) give uncertainties on the luminosities of individual clusters, for a cluster catalog that was constructed in a similar way to the maxBCG cluster catalog. These uncertainties are $\approx 10 \%$, and include both statistical and systematic effects. As discussed above, the SN Ia rate analysis includes 71 and 492 clusters from the $\mathrm{C} 4$ and maxBCG catalogs, respectively. If the uncertainties on the individual clusters are $\approx 10 \%$ and are independent, then the uncertainty on the total cluster luminosities will be $\lesssim 1 \%$. We conclude that uncertainty on the cluster luminosities is likely to be negligible in comparison to the statistical uncertainty on the cluster SN Ia rate measurement, but we acknowledge that any additional uncertainties on the cluster luminosities, or correlations between the uncertainties on the luminosity of the individual clusters, would impact the precision of our cluster SN Ia rate measurements.

\section{THE CLUSTER SN SAMPLE}

\subsection{Type Ia Supernovae}

To define the $\mathrm{SN}$ sample that is associated with galaxy clusters it is necessary to define selection criteria on both the SN lightcurve properties, and the $\mathrm{SN}$ spatial correlation with clusters. As was done in our study of the low-redshift SN rate from the first season of the SDSS-II Supernova Survey (Dilday et al. 2008), the $\mathrm{SNe}$ in the rate sample are required to meet certain selection criteria on their light-curve fits to the MLCS2k2 light curve model (Jha et al. 2007). For the present analysis the selection criteria are as follows:

1. At least 1 observation at $<-2$ days relative to peak in the supernova rest-frame.

2. At least 1 observation at $>t_{\text {late }}$ days relative to peak in the supernova rest-frame.

For maxBCG cluster SN candidates $t_{\text {late }}=10$ days, and for $\mathrm{C} 4$ cluster $\mathrm{SN}$ candidates $t_{\text {late }}=5$ days. The peak of the $\mathrm{SN}$ light curve is defined as the epoch of maximum luminosity in the rest-frame $B$ band.

3. Fit probability $>10^{-3}$.

The fit probability is computed assuming that the value of the minimum $\chi^{2}$ from the MLCS2k2 light curve fit follows a $\chi^{2}$ distribution with $N_{p}-4$ degrees of freedom, with $N_{p}$ the number of photometric observations. All SNe light curves are fit for epoch of maximum light, luminosity parameter $(\Delta)$, and extinction parameter $\left(A_{V}\right)$. When the SN redshift is known from spectroscopic measurement, the SN light curve is additionally fit for distance modulus. In the case of photometric $\mathrm{SNe}$, the additional fit parameter is the redshift of the $\mathrm{SN}$.
The requirement of a late-time photometric measurement is used primarily to reduce misidentification of photometric SN Ia candidates. For the low redshifts of the $\mathrm{C} 4$ galaxy clusters the majority of SNe have spectroscopic confirmation of type, and so a requirement of $t_{\text {late }}>5$ days increases the size of the $\mathrm{SN}$ sample, without introducing uncertainty from misidentification of photometric SNe. For the maxBCG clusters, the number of photometric cluster $\mathrm{SN}$ candidates is significant and a stricter requirement, $t_{\text {late }}>10$ days must be used. Additionally, the $\mathrm{SNe}$ that will be used for determining the $\mathrm{SN}$ rate are required to satisfy a set of selection criteria, based on fits of their search photometry to models of Type Ia, Type II, and Type Ib/c light curve models. These additional selection criteria are identical to those discussed in more detail in Dilday et al. (2010). Briefly, the SN candidates must be discovered in at least three epochs of the SN search, must fit the Type Ia model well in relation to the Type II and Type I/bc models, and must not have an overly broad light curve shape, as typified by the peculiar SN Ia 2005gj (Aldering et al. 2006; Prieto et al. 2007). The combination of cuts on light-curve sampling and shape effectively reject active galactic nuclei (AGNs) from the cluster SN Ia sample.

An SN candidate is defined to be associated with a galaxy cluster if it satisfies the following:

1. $\mathrm{SN}$ is within $1 \mathrm{Mpc} h^{-1}$ projected distance of the center of a cluster.

2. SN redshift is consistent with the cluster redshift.

Projected distance here refers to the orthogonal distance from the SN to the center of the galaxy cluster, assuming the redshift of the cluster. The choice of $1 \mathrm{Mpc} h^{-1}$ projected distance to define association with a cluster is chosen largely for consistency with previous cluster SN Ia rate measurements. The definition of redshift consistency depends on whether the SN and/or cluster redshifts are determined photometrically or spectroscopically. For the $\mathrm{C} 4$ clusters, the cluster redshifts are always precisely determined with a spectroscopic measurement, whereas the maxBCG cluster redshifts are determined photometrically. Consistency between the SN redshift, $z_{s}$, and cluster redshift, $z_{c}$, is defined in the following way:

1. Spectroscopic SN redshift and spectroscopic cluster redshift $\left|z_{s}-z_{c}\right|<0.015$.

2. Spectroscopic SN redshift and photometric cluster redshift $\left|z_{s}-z_{c}\right|<0.025$.

3. Photometric SN redshift and spectroscopic cluster redshift $\left|z_{s}-z_{c}\right|<2.5 \sqrt{(0.01)^{2}+\delta z_{s}^{2}}$.

4. Photometric SN redshift and photometric cluster redshift $\left|z_{s}-z_{c}\right|<2.5 \sqrt{(0.015)^{2}+\delta z_{s}^{2}}$,

where $\delta z_{s}$ is the error on the SN photometric redshift. In the case that both the SN and the cluster have spectroscopically determined redshifts, the allowable spread in galaxy redshifts is taken as 0.015 . This number was determined from the catalog of maxBCG cluster member galaxies. For each cluster that has at least four member galaxies with spectroscopically determined redshifts, we computed the rms of the difference between the member spectroscopic redshifts and the redshift assigned to the cluster. The mean of the resulting distribution of rms values is $\approx 0.015$. A similar value is obtained if the difference between the cluster redshift and the member galaxy spectroscopic redshifts are instead fit with Gaussian distributions, and the mean of the distribution of rms values for each Gaussian is computed. 
We note that for the nine $\mathrm{C} 4$ cluster $\mathrm{SNe}$ that have spectroscopic redshifts for both the SN and the cluster, the deviation in redshift is $\lesssim 0.002$ and this cut could be made more strict without affecting the $\mathrm{SN}$ rate result. In the case that the SN has a spectroscopically measured redshift and the cluster has a photometrically determined redshift (maxBCG clusters only) the tolerance on redshift consistency (0.025) is determined by adding in quadrature the rms spread of maxBCG member galaxy redshifts mentioned above (0.015) and the typical accuracy of the maxBCG cluster photometric redshifts $(\approx 0.015-0.020)$.

In the case that the $\mathrm{SN}$ redshift is determined photometrically, the cuts we apply correspond to a tolerance of $2.5 \sigma_{z}$, where $\sigma_{z}$ represents the error on the $\mathrm{SN}$ redshift, added in quadrature to a tolerance on the cluster redshift. This is a rather loose tolerance on redshift consistency, and we discuss this issue further in Section 5. There are $9 \mathrm{SNe}$ Ia in C4 clusters and $27 \mathrm{SNe}$ Ia from maxBCG clusters that satisfy the selection criteria, and these are listed in Table 3 (C4 clusters) and Table 4 (maxBCG clusters), respectively. We note that $\mathrm{SNe} 12979$ and 18375 occur in a cluster that is a member of both the $\mathrm{C} 4$ and maxBCG catalogs, and thus there are 34 distinct SNe Ia that pass the selection criteria. We note that SNe 16280, 18047, and 18362 are each associated with two distinct clusters, and that SNe 14279 and 16215 are associated with the same cluster. In the cases where a $\mathrm{SN}$ is associated with two clusters, we will count it only once in the $\mathrm{SN}$ rate calculation, assigning it to the nearest of the two clusters.

\section{CORRECTIONS TO THE SN Ia RATE MEASUREMENTS}

\subsection{Search Efficiency}

The method for determining the SN discovery efficiency is based on the same Monte Carlo (MC) studies discussed in Dilday et al. (2010). However, there is an important modification to be considered for $\mathrm{SNe}$ in galaxy clusters. It is a well established result that Type Ia SNe in early-type galaxies are more likely to be intrinsically faint, fast-declining SNe (e.g., Sullivan et al. 2006; Jha et al. 2007; M. Smith et al. 2010, in preparation). Therefore, the assumed distribution for the luminosity parameter of the MLCS2k2 light-curve model, $\Delta$, for the entire SN sample, as employed in Dilday et al. (2010), is not appropriate for considering SNe in galaxy clusters. To determine the SN discovery efficiency for SNe in galaxy clusters, we generated a set of $\mathrm{MC} \mathrm{SNe}$ with a $\Delta$ distribution similar to the observed distribution for cluster SNe. The $\Delta$ distribution assumed for cluster SNe Ia is shown in Figure 4. The fiducial $\Delta$ distribution has mean $\langle\Delta\rangle=0.033$, with an rms of 0.286 , and the cluster $\mathrm{SNe} \Delta$ distribution has mean $\langle\Delta\rangle=0.081$, with an rms of 0.246 . Assuming a larger mean value for $\Delta$ does not significantly affect the SN selection efficiency at the low redshifts of the $\mathrm{C} 4$ clusters, and has a $2 \%$ effect at the redshifts of the maxBCG clusters. The $\mathrm{SN}$ selection efficiency at the redshifts of the $\mathrm{C} 4$ clusters is approximately constant for each observing season, with the values $0.77,0.73$, and 0.72 for the 2005, 2006, and 2007 observing seasons, respectively. The redshift-dependent efficiencies used for the maxBCG cluster $\mathrm{SNe}$ are discussed in Dilday et al. (2010). Briefly, the efficiencies are well described by a function, $\epsilon_{0} /\left(1+e^{\left(z-z_{0}\right) / s_{z}}\right)$, with $\epsilon_{0} \approx 0.7$, $z_{0} \approx 0.35$, and $s_{z} \approx 0.05$. As in Dilday et al. (2008, 2010) the efficiency at low redshift is significantly less than 1 due mainly to the requirements of early and late time observing epochs.

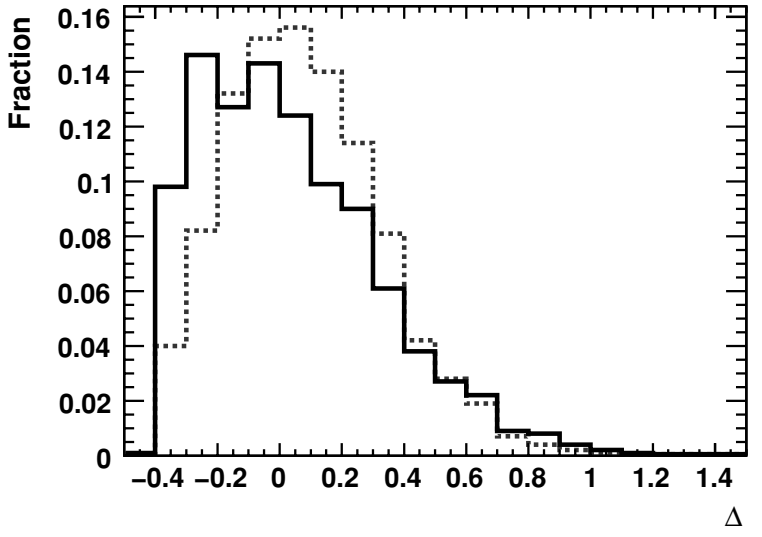

Figure 4. Assumed distribution for the MLCS2k2 $\Delta$ parameter for the entire SN sample (black/solid) and for the intrinsically fainter cluster SNe (gray/dashed).

\subsection{Contamination from Redshift Uncertainties}

In Section 4, we have defined a set of criteria on the consistency of the SN and cluster redshifts, which, in the case of photometric redshifts, corresponds roughly to a requirement that the redshifts match to within $\approx 2.5 \sigma$. We chose to apply a loose cut on the photometric redshift consistency because cluster $\mathrm{SNe}$ are rare by their nature and we wish to maximize the size of the sample, and because we assert that the requirement of a spatial correspondence with a cluster is already a strong filter on contamination from chance projections. Here we quantify further the expected contamination rate from chance projections, in the presence of finite error on the SN redshift measurement. We note that for the C4 cluster SN sample, all the SNe have spectroscopically measured redshifts (see Table 3), such that the contamination rate due to using $\mathrm{SN}$ photometric redshifts is manifestly 0 , and we focus exclusively on contamination in the maxBCG SN sample.

To estimate the expected contamination rate we employ the following procedure. For each cluster in the maxBCG catalog we compute the expected contamination,

$n_{\mathrm{contam}}^{i}=\int_{0}^{\infty} d z \eta(z) \int_{z_{\mathrm{cl}}-n_{\sigma} \sigma_{z}}^{z_{\mathrm{cl}}+n_{\sigma} \sigma_{z}} d z^{\prime} \frac{1}{\sqrt{2 \pi} \sigma_{z}} \exp \left(\frac{-\left(z-z^{\prime}\right)^{2}}{2 \sigma_{z}^{2}}\right)$,

where $\eta(z)$ is the SN number density, per unit redshift, $z_{\mathrm{cl}}$ is the cluster redshift, $\sigma_{z}$ is the assumed error on the $\mathrm{SN}$ photometric redshift, and $n_{\sigma}$ defines our cut on redshift consistency. The error on the photometric redshift, as a function of redshift, is shown in Dilday et al. (2010) to be $\sigma_{z} \approx 0.2 \times z^{1.5}$. To compute $\eta(z)$, we use the power-law rate model from Dilday et al. $\left(2008 ; r_{V}=2.6 \times 10^{-5}(1+z)^{1.5} \mathrm{SNe} \mathrm{Mpc}^{-3} h_{70}^{3}\right)$, along with the SDSS-II Supernova Survey SN efficiency function. The solid angle used in computing $\eta(z)$ is the solid angle corresponding to a $1 \mathrm{Mpc} h^{-1}$ separation from a cluster at redshift $z_{\mathrm{cl}}$. However, we assume that no SNe will be spatially associated with a cluster and have a redshift within \pm 0.015 of the cluster unless it is, in fact, associated with the cluster. Therefore, $\eta(z)$ is assumed to be 0 for $-0.015<z-z_{\mathrm{cl}}<+0.015$. The choice of 0.015 as the spread of maxBCG cluster redshifts is discussed in Section 4.1. For each cluster in the maxBCG catalog, we also compute the number of expected cluster SNe Ia, $N^{T}$, assuming a cluster SN Ia rate per unit $r$-band luminosity that is broadly consistent with those given by Sharon et al. (2007a) and Mannucci et al. (2008). The observed number of $\mathrm{SNe}$ that would be included in the 
Table 3

$\mathrm{SNe}$ in $\mathrm{C} 4$ Clusters

\begin{tabular}{|c|c|c|c|c|c|c|c|c|c|c|c|c|}
\hline SDSS Id & $\begin{array}{l}\text { IAU } \\
\text { Name }\end{array}$ & $\begin{array}{l}\mathrm{SN}^{\mathrm{a}} \\
\text { Type }\end{array}$ & $\begin{array}{c}\text { Cluster } \\
\text { R.A. (J2000) }\end{array}$ & $\begin{array}{c}\text { Cluster } \\
\text { Decl. (J2000) }\end{array}$ & $\begin{array}{l}\text { Cluster } \\
\text { Redshift }\end{array}$ & $\begin{array}{c}\text { SN } \\
\text { Redshift }\end{array}$ & $\begin{array}{c}\text { Projected } \\
\text { Dist. }\left(\mathrm{Mpc} h^{-1}\right)\end{array}$ & $N_{\text {gals }}$ & $u^{\mathrm{b}}$ & $r^{\mathrm{b}}$ & $u-r^{\mathrm{b}}$ & $r_{\mathrm{abs}}^{\mathrm{c}}$ \\
\hline 6295 & $2005 \mathrm{js}$ & Ia & 23.72890 & -0.65160 & 0.0814 & 0.0786 & 0.2804 & 38 & $19.268 \pm 0.052$ & $16.301 \pm 0.004$ & 2.967 & -21.663 \\
\hline 12979 & $2006 \mathrm{gf}$ & Ia & 11.56640 & +0.00200 & 0.1140 & 0.1153 & 0.1831 & 34 & $18.404 \pm 0.052$ & $15.438 \pm 0.003$ & 2.966 & -23.469 \\
\hline 13905 & $\ldots$ & Ia-photo+z & 11.54900 & +0.34870 & 0.1153 & 0.1151 & 0.7008 & 5 & $19.112 \pm 0.058$ & $16.224 \pm 0.004$ & 2.888 & -22.679 \\
\hline 14279 & $2006 \mathrm{hx}$ & Ia & 18.78670 & +0.29720 & 0.0450 & 0.0444 & 0.6849 & 128 & $18.179 \pm 0.027$ & $15.834 \pm 0.003$ & 2.345 & -20.782 \\
\hline 16215 & $2006 n e$ & $\mathrm{Ia}$ & 18.78670 & +0.29720 & 0.0450 & 0.0455 & 0.8912 & 128 & $19.532 \pm 0.088$ & $16.766 \pm 0.007$ & 2.766 & -19.907 \\
\hline $16280^{\mathrm{d}}$ & $2006 \mathrm{nz}$ & Ia & 14.27050 & -0.91880 & 0.0443 & 0.0370 & 0.7514 & 13 & $19.347 \pm 0.045$ & $16.404 \pm 0.004$ & 2.943 & -19.793 \\
\hline $16280^{\mathrm{d}}$ & $2006 \mathrm{nz}$ & Ia & 14.06720 & -1.25510 & 0.0442 & 0.0370 & 0.1348 & 65 & $19.347 \pm 0.045$ & $16.404 \pm 0.004$ & 2.943 & -19.793 \\
\hline 18375 & $20071 \mathrm{~g}$ & Ia & 11.56640 & +0.00200 & 0.1140 & 0.1169 & 0.2683 & 34 & $20.365 \pm 0.081$ & $18.167 \pm 0.008$ & 2.198 & -20.775 \\
\hline 18890 & $2007 \mathrm{~mm}$ & Ia & 16.43170 & -0.84980 & 0.0669 & 0.0654 & 0.2968 & 5 & $20.849 \pm 0.159$ & $17.888 \pm 0.008$ & 2.961 & -19.636 \\
\hline 19155 & $2007 \mathrm{mn}$ & $\mathrm{Ia}$ & 31.01580 & +0.25880 & 0.0770 & 0.0760 & 0.9720 & 8 & $19.298 \pm 0.070$ & $16.961 \pm 0.006$ & 2.337 & -20.922 \\
\hline 19968 & $2007 \mathrm{ol}$ & Ia & 24.34470 & -0.44720 & 0.0558 & 0.0551 & 0.3693 & 19 & $18.800 \pm 0.037$ & $16.290 \pm 0.004$ & 2.510 & -20.829 \\
\hline
\end{tabular}

Notes. SDSS Id denotes internal candidate designation. The horizontal lines delineate SNe discovered during the three distinct observing seasons of the SDSS-II Supernova Survey.

a "Ia" refers to spectroscopically identified SN; see Zheng et al. (2008). "Ia-photo+Z" refers to photometrically identified SN with a spectroscopically measured host galaxy redshift; see Dilday et al. (2010).

${ }^{\mathrm{b}}$ Value has been $k$-corrected to the rest frame of the galaxy; see Section 3.3.2.

${ }^{c}$ Observer frame magnitudes for the SN host galaxy.

${ }^{\mathrm{d}} \mathrm{SN}$ does not satisfy the color-typing criteria. See Dilday et al. (2010). 
Table 4

SNe in maxBCG Clusters

\begin{tabular}{|c|c|c|c|c|c|c|c|c|c|c|c|c|c|}
\hline SDSS Id & $\begin{array}{l}\text { IAU } \\
\text { Name }\end{array}$ & $\begin{array}{l}\mathrm{SN}^{\mathrm{a}} \\
\text { Type }\end{array}$ & $\begin{array}{c}\text { Cluster } \\
\text { R.A. }(\mathrm{J} 2000)\end{array}$ & $\begin{array}{c}\text { Cluster } \\
\text { Decl. (J2000) }\end{array}$ & $\begin{array}{c}\text { Cluster } \\
\text { Redshift }\end{array}$ & $\begin{array}{c}\text { SN } \\
\text { Redshift }\end{array}$ & $\begin{array}{c}\mathrm{SN}^{\mathrm{c}} \\
\text { Redshift Err. }\end{array}$ & $\begin{array}{c}\text { Projected } \\
\text { Dist. }\left(\mathrm{Mpc} h^{-1}\right)\end{array}$ & $N_{200}$ & $u^{\mathrm{d}}$ & $r^{\mathrm{d}}$ & $u-r^{\mathrm{d}}$ & $r_{\mathrm{abs}}^{\mathrm{b}}$ \\
\hline 1008 & 2005il & Ia-photo+z & 28.17341 & +1.13440 & 0.2025 & 0.2251 & $\ldots$ & 0.8972 & 19 & $23.036 \pm 0.855$ & $19.707 \pm 0.030$ & 3.329 & -20.963 \\
\hline 1740 & $\ldots$ & Ia-photo+z & 5.34753 & -0.82581 & 0.1837 & 0.1661 & $\ldots$ & 0.6152 & 25 & $21.553 \pm 0.235$ & $18.485 \pm 0.012$ & 3.068 & -21.362 \\
\hline 1782 & $\ldots$ & Ia-photo & 337.85896 & +0.29531 & 0.2781 & 0.2382 & 0.0189 & 0.5092 & 12 & $22.360 \pm 0.731$ & $17.682 \pm 0.008$ & 4.678 & -23.146 \\
\hline 5549 & $2005 \mathrm{hx}$ & Ia & 3.21852 & +0.22231 & 0.1405 & 0.1198 & $\ldots$ & 0.2569 & 16 & $21.847 \pm 0.347$ & $20.832 \pm 0.076$ & 1.015 & -18.172 \\
\hline 5717 & 2005 ia & Ia & 17.95342 & -0.01817 & 0.2646 & 0.2506 & $\ldots$ & 0.6039 & 16 & $22.807 \pm 0.456$ & $21.712 \pm 0.098$ & 1.095 & -19.258 \\
\hline 8280 & $\cdots$ & Ia-photo+z & 8.59684 & +0.85725 & 0.1972 & 0.1838 & $\ldots$ & 0.5378 & 32 & $19.844 \pm 0.122$ & $18.328 \pm 0.020$ & 1.516 & -21.789 \\
\hline 12979 & $2006 \mathrm{gf}$ & Ia & 11.60084 & +0.00238 & 0.1189 & 0.1153 & $\ldots$ & 0.0069 & 23 & $18.404 \pm 0.052$ & $15.438 \pm 0.003$ & 2.966 & -23.469 \\
\hline 13073 & $\ldots$ & Ia-photo & 335.99616 & +0.10358 & 0.2889 & 0.3246 & 0.0336 & 0.5282 & 15 & $\ldots$ & $\ldots$ & $\ldots$ & \\
\hline 13655 & $2006 \mathrm{hs}$ & Ia & 39.03651 & -1.00655 & 0.2727 & 0.2512 & $\ldots$ & 0.2146 & 13 & $21.435 \pm 0.264$ & $18.647 \pm 0.014$ & 2.788 & -22.330 \\
\hline 14340 & $\ldots$ & Ia-photo+z & 345.81978 & -0.85395 & 0.2754 & 0.2762 & $\ldots$ & 0.0741 & 37 & $23.409 \pm 1.323$ & $18.465 \pm 0.012$ & 4.944 & -22.783 \\
\hline 15201 & $2006 \mathrm{ks}$ & Ia & 337.53267 & -0.00373 & 0.2188 & 0.2073 & $\ldots$ & 0.1352 & 32 & $21.382 \pm 0.369$ & $18.175 \pm 0.013$ & 3.207 & -22.268 \\
\hline 15823 & $\ldots$ & Ia-photo+z & 314.20449 & +0.25007 & 0.2296 & 0.2142 & $\ldots$ & 0.6484 & 22 & $21.944 \pm 0.439$ & $19.248 \pm 0.023$ & 2.696 & -21.285 \\
\hline 16021 & $2006 \mathrm{nc}$ & Ia & 13.84586 & -0.33626 & 0.1459 & 0.1231 & $\ldots$ & 0.3384 & 15 & $20.585 \pm 0.171$ & $18.618 \pm 0.018$ & 1.967 & -20.455 \\
\hline 16467 & $\ldots$ & Ia-photo+z & 328.59792 & +0.08437 & 0.2106 & 0.2188 & $\cdots$ & 0.3120 & 21 & $22.307 \pm 0.544$ & $18.673 \pm 0.013$ & 3.634 & -21.918 \\
\hline 17435 & $2007 \mathrm{ka}$ & Ia & 20.36507 & +0.02192 & 0.2160 & 0.2210 & $\ldots$ & 0.3713 & 18 & $\ldots$ & $\ldots$ & $\cdots$ & \\
\hline 17568 & $2007 \mathrm{~kb}$ & Ia & 313.04022 & +0.33160 & 0.1486 & 0.1390 & $\ldots$ & 0.5406 & 23 & $22.017 \pm 0.424$ & $19.651 \pm 0.032$ & 2.366 & -19.732 \\
\hline 18047 & $\ldots$ & Ia-photo & 22.02004 & -0.66489 & 0.2592 & 0.3614 & 0.0446 & 0.5526 & 16 & $22.691 \pm 0.734$ & $19.566 \pm 0.023$ & 3.125 & -22.474 \\
\hline 18047 & $\cdots$ & Ia-photo & 22.08698 & -0.70110 & 0.2970 & 0.3614 & 0.0446 & 0.5014 & 15 & $22.691 \pm 0.734$ & $19.566 \pm 0.023$ & 3.125 & -22.474 \\
\hline 18362 & $\cdots$ & Ia-photo & 10.13752 & -0.23279 & 0.2350 & 0.2197 & 0.0278 & 0.4781 & 16 & $22.108 \pm 0.346$ & $19.446 \pm 0.023$ & 2.662 & -21.157 \\
\hline 18362 & $\cdots$ & Ia-photo & 10.06133 & -0.12293 & 0.2269 & 0.2197 & 0.0278 & 0.8767 & 28 & $22.108 \pm 0.346$ & $19.446 \pm 0.023$ & 2.662 & -21.157 \\
\hline 18375 & $2007 \mathrm{lg}$ & Ia & 11.60084 & +0.00238 & 0.1189 & 0.1169 & $\ldots$ & 0.4614 & 23 & $20.365 \pm 0.081$ & $18.167 \pm 0.008$ & 2.198 & -20.775 \\
\hline 18767 & $\ldots$ & Ia-photo & 4.55214 & +0.80302 & 0.2377 & 0.2728 & 0.0322 & 0.1752 & 13 & $22.471 \pm 0.656$ & $19.118 \pm 0.023$ & 3.353 & -22.094 \\
\hline 18909 & $2007 \mathrm{lq}$ & Ia? & 5.79401 & +0.97633 & 0.2296 & 0.2258 & $\ldots$ & 0.1231 & 16 & $21.935 \pm 0.534$ & $18.424 \pm 0.013$ & 3.511 & -22.255 \\
\hline 19001 & $\ldots$ & Ia-photo & 41.65704 & -0.38006 & 0.2457 & 0.2688 & 0.0243 & 0.7061 & 11 & $\ldots$ & $\ldots$ & $\ldots$ & \\
\hline 19341 & $2007 \mathrm{nf}$ & Ia & 15.84750 & +0.31945 & 0.2539 & 0.2339 & $\ldots$ & 0.1744 & 22 & $21.654 \pm 0.232$ & $18.875 \pm 0.014$ & 2.779 & -21.902 \\
\hline 19969 & $2007 \mathrm{pt}$ & Ia & 31.88020 & -0.24733 & 0.1593 & 0.1744 & $\cdots$ & 0.5710 & 15 & $19.966 \pm 0.067$ & $18.419 \pm 0.011$ & 1.547 & -21.558 \\
\hline 20111 & $2007 \mathrm{pw}$ & Ia & 354.47760 & +0.22478 & 0.2619 & 0.2468 & $\cdots$ & 0.8811 & 37 & $23.019 \pm 1.046$ & $19.321 \pm 0.024$ & 3.698 & -21.606 \\
\hline 20232 & $\cdots$ & Ia-photo+z & 7.08765 & -0.01711 & 0.2323 & 0.2154 & $\cdots$ & 0.3849 & 21 & $21.814 \pm 0.369$ & $18.592 \pm 0.012$ & 3.222 & -21.956 \\
\hline 20882 & $\ldots$ & Ia-photo & 16.97586 & +0.51513 & 0.2808 & 0.3181 & 0.0186 & 0.5792 & 19 & $24.582 \pm 1.345$ & $20.573 \pm 0.042$ & 4.009 & -21.086 \\
\hline
\end{tabular}

Notes. SDSS Id denotes internal candidate designation. The horizontal lines delineate SNe discovered during the three distinct observing seasons of the SDSS-II Supernova Survey.

a "Ia" and "Ia?" refer to spectroscopically identified SNe; see Zheng et al. (2008). "Ia-photo+z" and "Ia-photo" refer to photometrically identified SNe with and without a spectroscopically measured host galaxy redshift, respectively; see Dilday et al. (2010).

${ }^{\mathrm{b}}$ Value has been $k$-corrected to the rest frame of the galaxy; see Section 3.3.2.

${ }^{c}$ Errors are shown for photometric SNe Ia. For spectroscopically measured redshifts, the error is negligible and is not listed (Section 2).

${ }^{\mathrm{d}}$ Observer frame magnitudes for the SN host galaxy. 
sample, $N^{O}$, is then related to the true number, $N^{T}$, by

$$
\frac{N^{O}}{N^{T}}=\operatorname{erf}\left(n_{\sigma} / \sqrt{(2)}\right)+\frac{N_{\text {contam }}}{N^{T}},
$$

where $\operatorname{erf}(\cdot)$ is the error function, and $N_{\text {contam }}$ is the total expected contamination, $\Sigma_{i} n_{\text {contam }}^{i}$. To clarify our approach, we note that the right-hand side of Equation (13) can be computed, with reasonable assumptions about the value of the cluster SN Ia rate. We can then compare this to the observed value of the left-hand side of Equation (13), and thereby infer the contamination rate of the SN Ia sample due to finite redshift uncertainties. For cuts on redshift consistency of $n_{\sigma}=1.5$ and $n_{\sigma}=2.5, N_{\text {contam }} / N^{T}$ has the values $0.151\left(r_{\mathrm{cl}} / \mathrm{SNur}\right)^{-1}$ and $0.276\left(r_{\mathrm{cl}} / \mathrm{SNur}\right)^{-1}$, where $r_{\mathrm{cl}}$ is the assumed value of the cluster $\mathrm{SN}$ rate, and SNur $=\left(10^{10} L_{\odot}^{r}\right)^{-1}(100 \mathrm{yr})^{-1}$.

We first note that using values for $n_{\sigma} \approx 1.5-2.5$, we consistently arrive at $N^{T} \approx 5$. By way of example, if we choose $n_{\sigma}=2.5$ and $r_{\mathrm{cl}}=0.6 \mathrm{SNur}$, we then find $N^{O} / N^{T}=1.44$ (the right-hand side of Equation (13)). There are seven SNe Ia with photometrically determined redshifts that are within $\pm 2.5 \sigma$ of the cluster redshift (Table 3), and so this implies a value of $N^{T}=N^{O} / 1.44 \approx 5$ (the left-hand side of Equation (13)). Therefore, we apply a bias correction to our maxBCG SN sample by assuming that the number of photometric $\mathrm{SNe}$ in the sample is five, rather than the seven listed in Table 4. To estimate the systematic error on the size of the maxBCG SN sample, we vary the assumed power-law SN rate model according to the errors given in Dilday et al. (2008), and vary the assumed cluster rate as $r_{\mathrm{cl}}=(0.6 \pm 0.2)$ SNur, which results in an estimated systematic error of $\approx(-2$ to +3$) \%$ on the maxBCG cluster SN rate measurements, due to employing photometric redshifts for a subset of the cluster $\mathrm{SNe}$.

\subsection{Cluster Incompleteness and Contamination}

Here we discuss the effect of incompleteness and contamination in the galaxy cluster catalogs on our cluster SN rate measurements, and on our comparisons of the cluster $\mathrm{SN}$ rate to the rate in early-type galaxies in the field. In what follows we assume that the SN rate in both galaxy clusters and the field is proportional to the host luminosity, with proportionality constants $\alpha_{c}$ and $\alpha_{f}$ for clusters and the field, respectively. We note that the $\mathrm{C} 4$ cluster catalog has $\approx 10 \%$ incompleteness and an $\approx 5 \%$ contamination rate (Miller et al. 2005), and that the maxBCG catalog has $\approx 10 \%$ incompleteness and an $\approx 10 \%$ contamination rate (Koester et al. 2007b).

Cluster incompleteness. With the ansatz that the cluster SN Ia rate is proportional to the stellar luminosity, independent of cluster richness, cluster incompleteness does not affect the derived value of the $\mathrm{SN}$ rate. That is, although real clusters may fail to be included in the cluster catalog, the cluster SN sample will be reduced in direct proportion to the luminosity of the missing clusters. Cluster incompleteness, however, will affect the value of the ratio of the rates for cluster versus field earlytype galaxies, as $\mathrm{SNe}$ occurring in clusters may be erroneously associated with the field SN sample.

In the presence of cluster incompleteness, the inferred ratio of the SN Ia rate in clusters, $r_{c}$, to the SN rate in field early-type galaxies, $r_{f}$, will be

$$
\frac{r_{c}}{r_{f}}=\left(\frac{N_{c}-N_{\delta-}}{L_{c}-L_{\delta-}}\right)\left(\frac{L_{f}+L_{\delta-}}{N_{f}+N_{\delta-}}\right)
$$

$$
\approx \frac{\alpha_{c}}{\alpha_{f}}\left(1+\left(1-\alpha_{c} / \alpha_{f}\right)\left(L_{\delta-} / L_{c}\right)\left(L_{c} / L_{f}\right)\right),
$$

where $N(L)$ represents the true number of $\mathrm{SNe}$ (luminosity) in a given subset, and the $c, f$, and $\delta$ - subscripts denote clusters, the field, and the portion of the clusters missed due to incompleteness, respectively. The quantity $L_{\delta-} / L_{c}$ is the cluster incompleteness. To estimate the quantity $L_{c} / L_{f}$, we compute a cluster luminosity density by dividing the summed $r$-band luminosity from the maxBCG cluster catalog $(k$-corrected from ${ }^{0.25} i$ as described in Section 3.3), for clusters in the SN survey region, by the corresponding volume. For redshifts $z \gtrsim 0.2$ this derived cluster luminosity density has the value $\approx 0.104 \times$ $10^{8} L_{\odot} \mathrm{Mpc}^{-3} h$. In Dilday et al. (2008), we showed that, using the LFs of Blanton et al. (2003b), and an objective classification scheme for early-type galaxies, the ${ }^{0.1} r$-band luminosity density, for early-type galaxies is $0.994 \times 10^{8} L_{\odot} \mathrm{Mpc}^{-3} h$. Applying a $k$-correction as described in Section 3.3, this corresponds to a $r$-band luminosity density, for early-type galaxies, of $1.24 \times 10^{8} L_{\odot} \mathrm{Mpc}^{-3} h$. Therefore, we conclude that the ratio of the total $r$-band light in cluster galaxies versus field early-type galaxies, $L_{c} / L_{f}$, is $\approx 0.091$. The fraction of cluster luminosity contained in early-type galaxies is $\approx 80 \%$ (Hansen et al. 2009), and thus the ratio of the $r$-band light in cluster early-type galaxies versus field early-type galaxies, $\left(L_{c} / L_{f}\right)_{\text {red }}$, is $\approx 0.073$. In turn, this results in an estimated $\approx-(1-2) \%$ correction factor, for values of the ratio of the $\mathrm{SN}$ rates, $\alpha_{c} / \alpha_{f}$, of $\approx 2-3$.

Cluster contamination. Contamination of the cluster catalogs will affect both the value of the SN rate in clusters, and the ratio of the rate in clusters to the rate in the field.

The inferred cluster $\mathrm{SN}$ rate will be

$$
\begin{aligned}
r_{c} & =\frac{N_{c}+N_{\delta+}}{L_{c}+L_{\delta+}} \\
& \approx \alpha_{c}\left(1+\left(\alpha_{f} / \alpha_{c}-1\right) L_{\delta+} / L_{c}\right),
\end{aligned}
$$

where $N$ and $L$ are defined as in Equation (14), and $\delta+$ denotes field quantities that were erroneously identified with clusters. The quantity $L_{\delta+} / L_{c}$ represents the cluster contamination rate. The estimated correction factor on the SN Ia cluster rate measurement due to cluster contamination is then $\approx+3 \%$ for $\mathrm{C} 4$ clusters, and $\approx+(5-7) \%$ for maxBCG clusters.

The inferred ratio of the $\mathrm{SN}$ rate will be

$$
\begin{aligned}
\frac{r_{c}}{r_{f}} & =\left(\frac{N_{c}+N_{\delta+}}{L_{c}+L_{\delta+}}\right)\left(\frac{L_{f}-L_{\delta+}}{N_{f}-N_{\delta+}}\right) \\
& \approx \frac{\alpha_{c}}{\alpha_{f}}\left(1+\left(\alpha_{f} / \alpha_{c}-1\right) L_{\delta+} / L_{c}\right),
\end{aligned}
$$

with estimated correction factors on the cluster versus field SN rates of $\approx+3 \%$ for $\mathrm{C} 4$ clusters, and $\approx+(5-7) \%$ for maxBCG clusters.

\subsection{Summary of SN Ia Rate Corrections}

To summarize our consideration of necessary corrections to our cluster SN Ia rate measurements, we have considered the effects of the lesser average intrinsic luminosity for SNe Ia in early-type galaxies as compared to a representative galaxy sample, contamination of the cluster SN Ia sample due to the use of 
SN photometric redshifts, and incompleteness (clusters are not identified by the cluster finding algorithms) and contamination (objects that are not clusters are identified as clusters) of the galaxy cluster catalogs. The lesser average intrinsic luminosity of SNe Ia in early-type galaxies does not affect the selection efficiency for the relatively low-redshift $\mathrm{C} 4 \mathrm{SN}$ sample, and has an $\approx 2 \%$ effect on the maxBCG SN sample. This factor is accounted for through the efficiency function, $\epsilon(z)$, that is mentioned in Section 7 . We estimate that $\approx 2$ of the $7 \mathrm{SNe}$ with photometric redshifts in the maxBCG SN Ia sample are not in fact associated with the cluster, and thus we will apply a correction of $f^{p z} \approx-2 / 27=-7.4 \%$ to the maxBCG cluster rate. Cluster contamination results in correction factors of $f^{c} \approx+3 \%$ for the $\mathrm{C} 4$ cluster $\mathrm{SN}$ Ia rate, and $f^{c} \approx+(5-7) \%$ for the maxBCG cluster $\mathrm{SN}$ Ia rate. Cluster contamination also results in correction factors on the ratio of the $\mathrm{SN}$ Ia rate in early-type cluster galaxies to early-type field galaxies of $f^{c} \approx+3 \%$ for the $\mathrm{C} 4$ clusters, and $f^{c} \approx+(5-7) \%$ for the maxBCG clusters. Additionally, cluster incompleteness results in an $f^{I} \approx-(1-2) \%$ correction factor on the ratio of the $\mathrm{SN}$ Ia rate in early-type cluster galaxies to early-type field galaxies for both the $\mathrm{C} 4$ and the maxBCG clusters. We note that in Dilday et al. (2010) it is shown that the contamination of the SDSS-II SN Survey photometric SN Ia sample by non-Ia $\mathrm{SNe}$ is not more than $\approx 3 \%$. Photometric $\mathrm{SNe}$ Ia make up $\approx 50 \%$ of the maxBCG cluster SN Ia sample, and so the overall contamination of the cluster SN Ia sample is $\lesssim 2 \%$. Contamination of the SN Ia sample due to lack of a spectroscopic identification is likely to be correlated with the contamination due to the use of photometric redshifts discussed above, and so the size of the effect is expected to be less than this.

\section{SYSTEMATIC ERRORS}

Here we discuss possible sources of systematic error on our cluster SN Ia rate measurements. In the previous section we derived correction factors to account for incompleteness and contamination of the galaxy cluster catalogs. These correction factors will each have an uncertainty due to sample variance in the galaxy clusters in the SN survey region. We evaluate the uncertainty by assuming that each galaxy cluster in the sample will suffer from incompleteness or contamination with a probability given by the mean incompleteness and contamination rates given above. The uncertainty on the correction factor is then derived from the variance of the corresponding binomial distribution, with the number of events equal to the number of galaxy clusters considered. For the C4 clusters (71 clusters), this results in $\mathrm{a} \pm 3.2 \%$ uncertainty on the correction for cluster incompleteness and a $\pm 2.5 \%$ uncertainty on the correction for cluster contamination. For the maxBCG clusters (492 clusters), this results in a $-1.2 \%$ to $+1.0 \%$ uncertainty on the correction for both cluster incompleteness and cluster contamination. Dilday et al. (2010) discuss the systematic uncertainty on the SN Ia rate for the full SDSS-II SN Survey sample, and show that assuming a value for the mean extinction in the $V$ band, $\left\langle A_{V}\right\rangle=0.45$, as opposed to the fiducial value of $\left\langle A_{V}\right\rangle=0.35$, has a large systematic effect on the derived SN Ia rates for SNe Ia at $z \gtrsim 0.2$. For the present sample of SNe Ia in galaxy clusters, assuming a larger than fiducial value for the mean dust extinction is not appropriate, and so we do not include this systematic effect on the cluster SN Ia rate measurements. As noted in Section 3.4, any uncertainty on the cluster luminosities will also contribute to the total uncertainty on the cluster SN Ia rate.

\section{SDSS SN RESULTS}

\subsection{The C4 Cluster Rate}

As discussed above, there are nine Type Ia SNe in C4 clusters that satisfy the SN selection criteria. The total $r$-band luminosity in the SN survey region, after correcting for the faint end of the LF, is $4.99 \times 10^{13} L_{\odot}^{r} h^{-2}$, and the mean redshift of the C4 clusters is $\langle z\rangle=0.0786$. The observing time for the SDSS-II Supernova Survey was 89, 90, and 90 days, for the 2005, 2006, and 2007 observing seasons, respectively. The SN selection efficiency is approximately constant over the range of the $\mathrm{C} 4$ clusters, with the values $0.77,0.73$, and 0.72 for the 2005,2006 , and 2007 observing seasons, respectively. Using these values, the $\mathrm{SN}$ rate in $\mathrm{C} 4$ clusters is

$$
r=\frac{N(1+\langle z\rangle) g^{c}}{\Sigma L_{r} \Sigma_{i}(\epsilon T)}=0.37_{-0.12-0.01}^{+0.17+0.01} \mathrm{SNu} r h^{2},
$$

where $i$ denotes each observing season, $T$ is the survey observation time, $\epsilon$ is the $\mathrm{SN}$ selection efficiency, $N$ is the number of SNe Ia, $\Sigma L_{r}$ is the total cluster luminosity in the $r$ band, and $\mathrm{SNu} r=\left(10^{10} L_{\odot}^{r}\right)^{-1}(100 \mathrm{yr})^{-1}$. The factor $g^{c}=1+f^{c}=1.03$, where $f_{c}$ is the correction due to contamination of the cluster catalog, discussed in Section 5. The errors quoted are the $1 \sigma$ statistical and systematic errors, respectively. We have used the mean survey efficiency, determined from Monte Carlo simulations, as both the positions of the clusters and the Monte Carlo SNe are effectively uniform random samplings of the survey area. Jorgensen (1997; clusters) and Padmanabhan et al. (2004; field) give the average stellar mass to luminosity ratio ( $r$-band) for early-type galaxies as $\approx 3$, which is in good agreement with the $M / L$ conversion employed by Sharon et al. (2007a). Using this assumption, the Type Ia SN rate per unit luminosity quoted above is equal to $0.123_{-0.040-0.003}^{+0.056+0.004} \mathrm{SNuM} h^{2}$, with $\mathrm{SNuM}=\left(10^{10} M_{\odot}\right)^{-1}(100 \mathrm{yr})^{-1}$. Using the same average conversion factor from $r$ to $B$ as Sharon et al. (2007a), this corresponds to $0.46_{-0.15-0.01}^{+0.21+0.01} \mathrm{SNu} B h^{2}$.

\subsection{The maxBCG Cluster Rate}

As discussed in Section 4, there are 27 SNe in maxBCG clusters from SDSS-II Supernova Survey that satisfy the selection criteria. As the SDSS-II Supernova Survey SN discovery efficiency is not well approximated as constant over the redshift range of the maxBCG catalog, in determining the $\mathrm{SN}$ rate in maxBCG clusters we use the more formal definition of the SN rate per unit luminosity, $r_{L}$,

$$
r_{L}=\frac{N g^{p z} g^{c}}{\widetilde{\epsilon T L L}}
$$

with

$$
\widetilde{\epsilon T \Sigma L}=T_{\oplus} \int_{z_{\min }}^{z_{\max }} d z \frac{\Sigma L(z) \epsilon(z)}{1+z},
$$

where $T_{\oplus}$ is the earth frame observation time, $\Sigma L(z)$ is the total cluster luminosity as a function of redshift, and $\epsilon(z)$ is the SN discovery efficiency. The factors $g^{p z}$ and $g^{c}$ represent the corrections due to use of $\mathrm{SN}$ photometric redshifts and contamination of the cluster catalog, respectively (Section 5). These factors have the values $g^{p z}=1+f^{p z}=0.926$ and $g^{c}=1+f^{c}=1.06$. For the maxBCG catalog $z_{\min }$ is fixed to 0.1 . In Figure 5, we show the value of the maxBCG cluster SN rate as a function of $z_{\max }$. This figure shows that the derived 


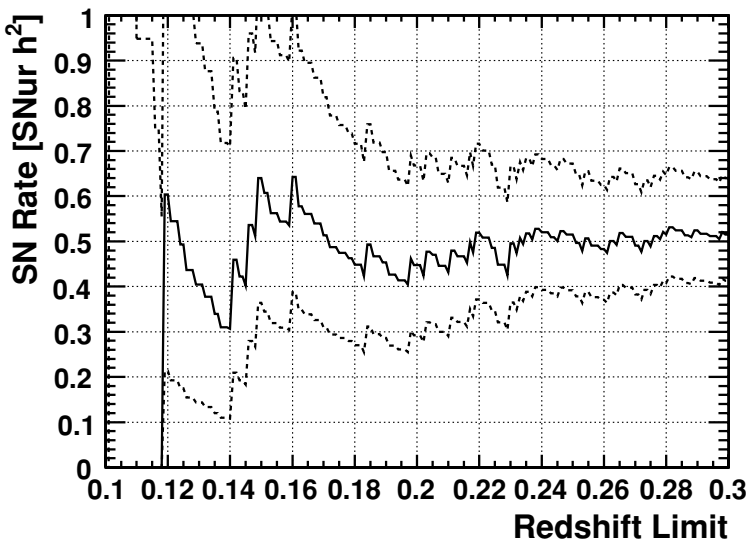

Figure 5. maxBCG cluster $\mathrm{SN}$ rate as a function of upper limit on the redshift range. The dashed lines represent the $1 \sigma$ upper and lower limit of the $\mathrm{SN}$ rate.

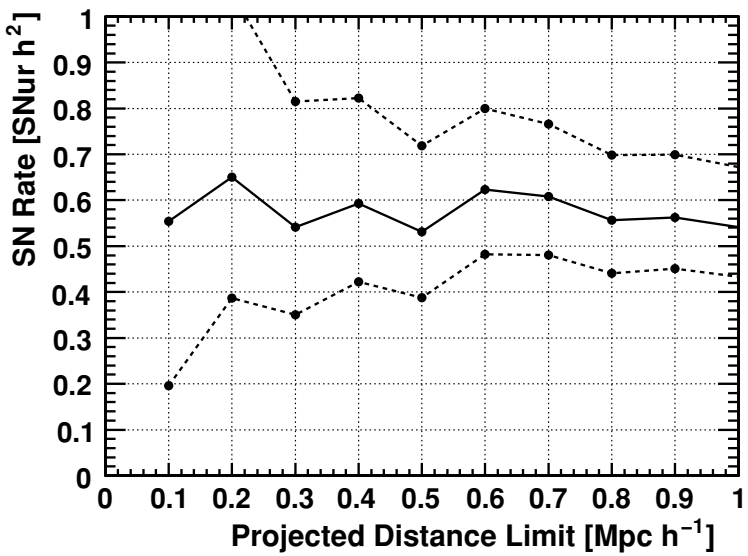

Figure 6. maxBCG cluster $\mathrm{SN}$ rate as a function of limit on the projected distance. The dashed lines represent the $1 \sigma$ upper and lower limit of the SN rate.

SN rate is not strongly sensitive to the upper limit on the cluster sample. If the upper limit is chosen as the upper limit of the maxBCG catalog, $z=0.3$, then we have $N=27$ and $\widetilde{\epsilon T \Sigma L}=4.85 \times 10^{13} \mathrm{yr} L_{\odot}^{r} h^{-2}$, including the correction for the faint end of the LF. The derived value of the SN rate is thus $r_{L}=0.55_{-0.11-0.01}^{+0.13+0.02} \mathrm{SNu} h^{2}\left(=0.68_{-0.14-0.02}^{+0.17+0.02} \mathrm{SNu} B h^{2}=\right.$ $\left.0.18_{-0.036-0.004}^{+0.044+0.006} \mathrm{SNu} M h^{2}\right)$. Figure 6 shows the cluster $\mathrm{SN}$ rate as a function of the limit on the projected distance from the center of the cluster, in units of $\mathrm{Mpc} h^{-1}$. The fraction of red, earlytype galaxies in clusters is larger at small separations from the cluster center, and in this sense the $\mathrm{SN}$ rate at smaller separations is a more reliable probe of the component of the $\mathrm{SN}$ rate that originates from an old stellar population. Furthermore, the extent of a cluster is not an unambiguously defined quantity, and $\mathrm{SNe}$ at smaller separations are more robustly associated with the cluster. Figure 6 shows that the derived SN rate is not strongly dependent on the limiting projected radius that we use to define cluster membership. Figure 7 shows the SN rate as a function of the lower limit on the cluster richness measure, $N_{200}$.

The SDSS-II Supernova Survey cluster rate results, along with the previous measurements listed in Table 1, in units of $\mathrm{SNu} B h^{2}$, are shown in Figure 8. A fit of the data to a linear model of the cluster SN Ia rate as a function of redshift, $r=A+B z$, gives best fit values of $A=0.49 \pm 0.14 \mathrm{SNu} B$ $h^{2}$ and $B=0.81_{-0.80}^{+0.82}\left(\chi^{2} / \mathrm{NDF}=0.88 / 5\right)$. A fit of the data to a constant model of the cluster SN Ia rate as a function of

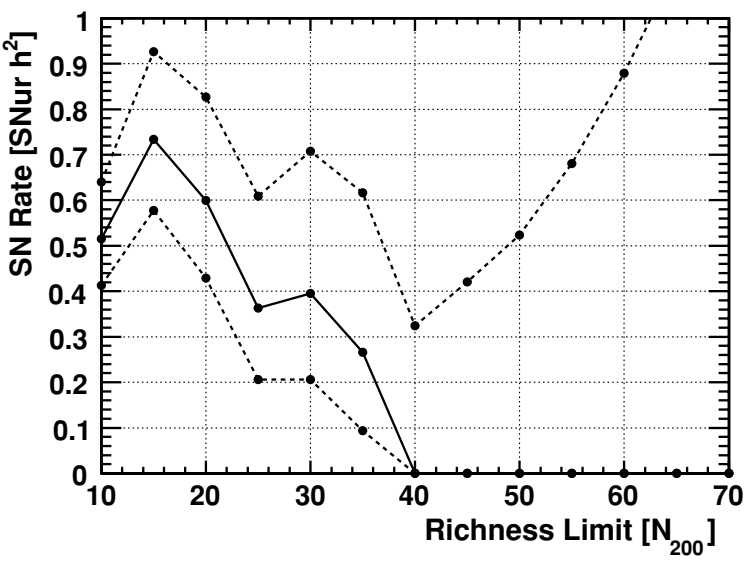

Figure 7. maxBCG cluster SN rate as a function of lower limit on the cluster richness measure, $N_{200}$. The dashed lines represent the $1 \sigma$ upper and lower limit of the $\mathrm{SN}$ rate.

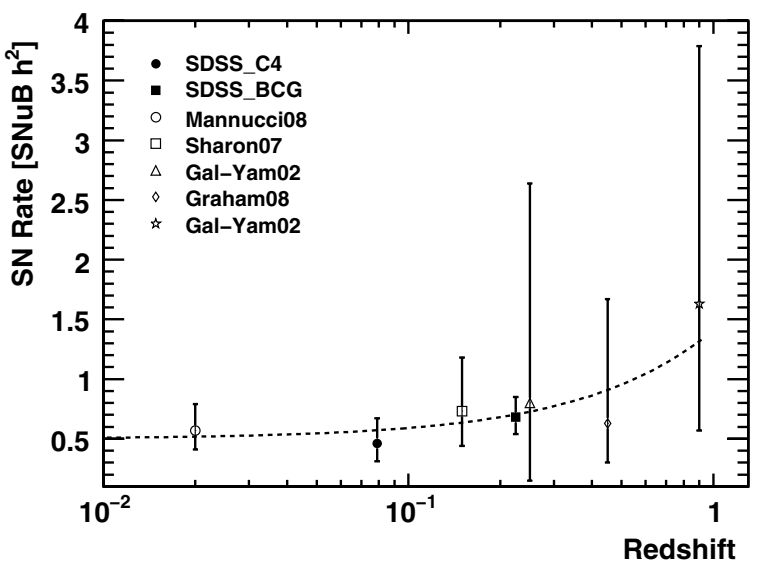

Figure 8. Cluster SN Ia rate vs. redshift. The dashed line shows the best fit to a linear model of the $\mathrm{SN}$ rate as a function of redshift.

redshift, $r=A$, gives a best fit value of $A=0.61_{-0.89}^{+0.95} \mathrm{SNu} B h^{2}$ $\left(\chi^{2} / \mathrm{NDF}=1.9 / 6\right)$.

\subsection{Cluster SN Rate Versus Field SN Rate}

In Dilday et al. (2008), the SN rate in low-redshift earlytype galaxies was estimated from the first year data of the SDSS-II Supernova Survey as $\approx 0.17_{-0.04}^{+0.06} \mathrm{SNu} r h^{2}$. One SN in the sample described in Dilday et al. (2008) is a cluster SN, and hence the early-type field rate is $\approx 0.16_{-0.04}^{+0.06} \mathrm{SNu} r h^{2}$. Using the same color cut $(u-r=2.4)$ to differentiate early- and latetype galaxies as Dilday et al. (2008), we find that $6 / 9$ of the $\mathrm{C} 4$ cluster SNe reside in early-type galaxies, while 20/27 maxBCG cluster $\mathrm{SNe}$ reside in early-type galaxies (18/25 after bias correction to account for SN photometric redshifts). According to Hansen et al. (2009), the fraction of light in early-type galaxies for the maxBCG clusters is $\approx 80 \%$. Therefore, the cluster SN rate for early-type galaxies is $r_{L}=0.31_{-0.12-0.01}^{+0.18+0.01} \mathrm{SNu} r h^{2}$ and $r_{L}=0.49_{-0.11-0.01}^{+0.15+0.02} \mathrm{SNu} r h^{2}$ for $\mathrm{C} 4$ and maxBCG clusters, respectively. As discussed in Section 5, the ratio of the cluster early-type to field early-type rates must be multiplied by a factor $g^{I}=1-f^{I}=0.985$ to account for incompleteness of the galaxy cluster catalogs. The $\mathrm{C} 4$ and maxBCG cluster SN Ia rates are seen to be larger by factors of $1.94_{-0.91-0.015}^{+1.31+0.043}$ and $3.02_{-1.03-0.048}^{+1.31+0.062}$ compared to the field SN rate for earlytype galaxies, respectively. This is in broad agreement with the 

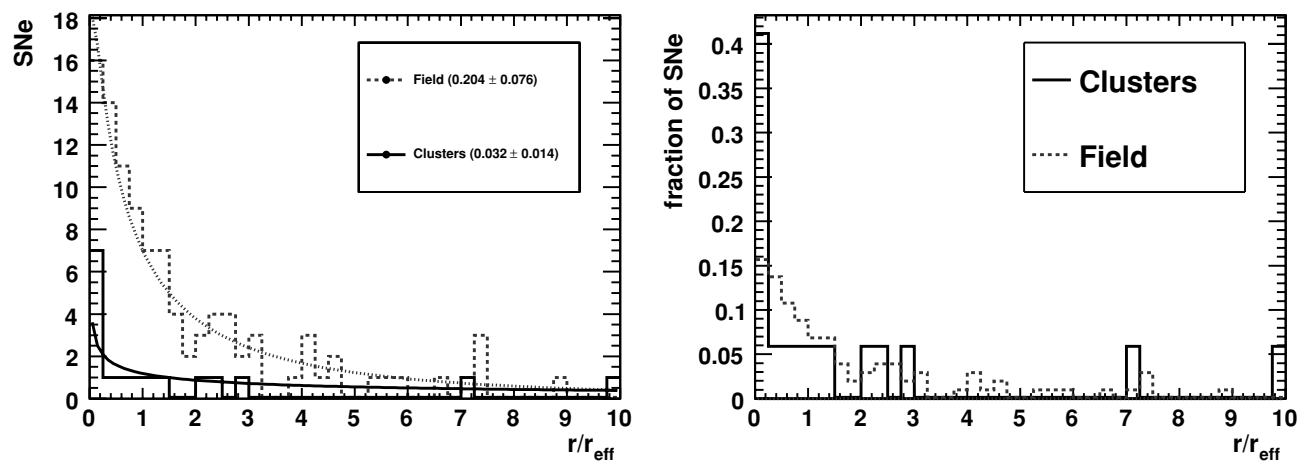

Figure 9. Radial distribution of $\mathrm{SNe}$ in early-type host galaxies.

enhancement of the Type Ia SN rate in early-type galaxies in galaxy clusters reported by Mannucci et al. (2008).

\subsection{The SN Ia Rate in Cluster BCGs}

Of the $32 \mathrm{SNe}$ in our cluster sample (after accounting for duplicates and for contamination due to use of SN photometric redshifts), two are in the BCG of the cluster. These are SNe 12979 and 13905. Another, SN 18890 appears to be in the BCG, although it has a somewhat ambiguous host. All three of these $\mathrm{SNe}$ are in $\mathrm{C} 4$ clusters, while one is in a maxBCG cluster. The cluster BCGs contain $\approx 6 \%$ of the total $r$-band cluster luminosity, and so the SN Ia rate, in the cluster BCGs, is $r_{L}=2.04_{-1.11-0.04}^{+1.99+0.07} \mathrm{SNu} h^{2}$ for the $\mathrm{C} 4$ clusters and $r_{L}=0.36_{-0.30-0.01}^{+0.84+0.01} \mathrm{SNu} r h^{2}$ for the maxBCG clusters.

\subsection{Hostless SNe}

For the SNe Ia in our cluster sample, all but two are visually associated with a host galaxy. The two SNe Ia with no host evident in the images are SN 13073 and SN 19001. Additionally, one SN, SN 1782, lies at a distance $\approx 25 \mathrm{kpc}^{-1}$ from its presumed host galaxy, and might reasonably be considered to be hostless. Assuming SN 1782 is in fact a hostless SN, we can constrain the fraction of hostless $\mathrm{SNe}$ in galaxy clusters to be $\approx 9.4 \%$, with $1 \sigma$ confidence interval $(4.3-17.7) \%$. For comparison, Gal-Yam et al. (2003) reported two hostless SNe from a sample of seven for an estimated fraction of hostless $\mathrm{SNe}$ of $\approx 30 \%$. We note, however, that the classification of these two SNe as hostless by Gal-Yam et al. (2003) relied, in part, on the observation of velocity offsets between the SNe and its potential host of $\approx 750-2000 \mathrm{~km} \mathrm{~s}^{-1}$. In principle a more thorough analysis of $\mathrm{SN}$ and galaxy spectra for $\mathrm{SNe}$ from the SDSS-II Supernova Survey may reveal additional hostless SNe. The clusters considered by Gal-Yam et al. (2003) were generally more massive than the clusters considered here, and an additional possibility is that the rate of hostless SNe Ia in galaxy clusters is larger for more massive clusters.

\subsection{Radial Distribution of Cluster SNe}

Forster \& Schawinski (2008) have studied the radial distribution of SNe in early-type galaxies. The sample of SNe includes an unspecified number of SNe from the SDSS-II Supernova Survey. Here we consider the radial distribution for $\mathrm{SNe}$ for field and cluster ellipticals.

To construct the sample of early-type galaxies, we employ the following procedure. We first assign a host galaxy to each $\mathrm{SNe}$ by locating the nearest host from the SDSS database, in units of the isophotal radius of the host galaxy in the $r$ band. The algorithm is described in detail in Dilday et al. (2008). Earlytype galaxies are defined as those host galaxies that satisfy the following criteria:

$$
\begin{aligned}
& \text { 1. } u-r>2.4 \text {, } \\
& \text { 2. } r<21.5 \text {, } \\
& \text { 3. } \Delta r<0.05 \text {, }
\end{aligned}
$$

where $u$ and $r$ are the SDSS model magnitudes and $\Delta r$ is the error on the $r$-band magnitude. It is a well established result that the $(u-r)$ color for SDSS galaxies is bimodal (Strateva et al. $2001)$, with early-type galaxies generally having $(u-r)>2.2$. As discussed in Dilday et al. (2008), studies of the observed $u-r$ distribution for galaxies from the photometric redshift catalog of Oyaizu et al. (2008) suggest that $u-r=2.4$ may provide a more robust separation. The requirement that $r<21.5$ is imposed as the separation of stars and galaxies is fairly robust to this limit. The requirement that $\Delta r<0.05$ is imposed to remove outlying, poorly measured galaxies. The distributions of the distance of each SN from its host galaxy, for field and cluster early-type hosts, is shown in Figure 9. For field early-type galaxies, a fit of the data to a Sersic model of the luminosity distribution, $d N / d r=A \rho e^{-\gamma \rho^{-\lambda}}$, where $\rho$ denotes the distance of the SN in units of the de Vaucouleurs radius of the host galaxy, gives a value for $\lambda$ of $0.20 \pm 0.08$, which is consistent with a de Vaucouleurs profile $(\lambda=0.25)$. Since the distribution of light in early-type galaxies is known to follow a de Vaucouleurs profile, the result of the fit confirms the results of Forster \& Schawinski (2008) that the SN rate in field ellipticals is well represented by a constant rate per unit luminosity. The radial distribution for $\mathrm{SNe}$ in cluster earlytype galaxies shows an enhancement at small radial separations in comparison to a de Vaucouleurs profile. An enhancement of the SN Ia rate in regions that have undergone recent star formation has been reported by several authors (see, e.g., the review by Mannucci 2009), and our observed enhancement at small radial separations is possible evidence for a component of the cluster SN Ia rate that tracks residual star formation activity in cluster early-type galaxies. Such an enhancement is in qualitative agreement with the larger SN Ia rate in cluster ellipticals, compared with field ellipticals, mentioned above and by Mannucci et al. (2008), as well as with recent independent evidence for some recent star formation activity in early-type galaxies (e.g., Kaviraj et al. 2007). Additionally, Della Valle et al. (2005) and Graham et al. (2010) report an enhancement of the SN Ia rate in radio-loud early-type galaxies, and attribute this to mergers that not only power the radio emission, but also provide a young stellar population that can account for the enhanced SN Ia rate. While we do not 
consider the radio properties of the early-type galaxies in our sample, it is possible that our results are related to the results of Della Valle et al. (2005) and Graham et al. (2010). While the best fit radial profile for cluster early-type galaxies does not match a de Vaucouleurs profile, a Kolmogorov-Smirnov (KS) test on the radial distributions of SNe Ia in cluster and field ellipticals results in an $\approx 30 \%$ probability that the data are drawn from the same underlying distribution. As discussed in Dilday et al. (2008), all SN selection efficiency calculations are based ultimately on artificial SNe Ia inserted directly into the survey search imaging data. Analysis of these artificial $\mathrm{SNe}$ Ia from the three observing seasons of the SDSS-II Supernova Survey does not show evidence for significant loss of efficiency near the cores of galaxies. While the artificial $\mathrm{SNe}$ were inserted into random galaxies that on the average may be less luminous in the cores than typical cluster galaxies, any additional inefficiency would only increase the observed enhancement of the SN Ia rate in the cores of cluster early-type galaxies.

\section{CONCLUSIONS}

We have presented measurements of the Type Ia SN rate in galaxy clusters over the redshift range $0.03<z<0.30$. These measurements are based on 34 SNe Ia (32 after applying a bias correction) from the SDSS-II Supernova Survey and represent a significant statistical contribution to the study of the Type Ia $\mathrm{SN}$ rate in galaxy clusters. Our results on the Type Ia SN rate are consistent with previously published measurements, both in the local universe, and at redshift $\approx 0.15-0.25$. In turn, the local and low-redshift SN cluster rates are consistent with the $\mathrm{SN}$ Ia rate at $z \approx 0.45$ and at $z \approx 0.9$. The current cluster SN Ia rate measurements do not show evidence for a rapid increase in the $\mathrm{SN}$ rate, as a function of lookback time, as has been well-established for the volumetric SN Ia rate. It should be emphasized, however, that the existing cluster SN rate measurements are based on small samples and the measurements do not rule out a redshift dependence to the cluster $\mathrm{SN}$ rate. A fit of the cluster SN Ia rate measurements to a linear dependence on redshift results in a best fit slope of $\left(0.91_{-0.81}^{+0.85}\right) \mathrm{SNu} B h^{2}$ per unit redshift. We find a ratio of the $\mathrm{SN}$ Ia rate in cluster early-type galaxies to that of the SN Ia rate in field early-type galaxies of $1.94_{-0.91-0.015}^{+1.31+0.043}$ and $3.02_{-1.03-0.048}^{+1.31+0.062}$, for C4 and maxBCG clusters, respectively.

We find that at most three of the $\mathrm{SNe}$ Ia in our sample are hostless, intra-cluster $\mathrm{SNe}$, which is significantly less than the $30 \%$ hostless fraction estimated from previous cluster SN studies.

We have presented the first study of the radial distribution of SNe Ia in cluster early-type galaxies. The radial distribution for $\mathrm{SNe}$ in cluster early-type galaxies shows an enhancement at small radial separations in comparison to the radial distribution in field early-type galaxies, which are well described by a de Vaucouleurs profile. This enhancement could be attributable to residual star formation in cluster early-type galaxies, which could explain the higher SN Ia rate observed in cluster early-type galaxies in comparison to early-type galaxies in the field.

Funding for the SDSS and SDSS-II has been provided by the Alfred P. Sloan Foundation, the Participating Institutions, the National Science Foundation, the U.S. Department of Energy, the National Aeronautics and Space Administration, the Japanese Monbukagakusho, the Max Planck Society, and the Higher Education Funding Council for England. The SDSS Web site is http://www.sdss.org/. The SDSS is managed by the Astrophysical Research Consortium for the Participating Institutions. The Participating Institutions are the American Museum of Natural History, Astrophysical Institute Potsdam, University of Basel, University of Cambridge, Case Western Reserve University, University of Chicago, Drexel University, Fermilab, the Institute for Advanced Study, the Japan Participation Group, Johns Hopkins University, the Joint Institute for Nuclear Astrophysics, the Kavli Institute for Particle Astrophysics and Cosmology, the Korean Scientist Group, the Chinese Academy of Sciences (LAMOST), Los Alamos National Laboratory, the Max-PlanckInstitute for Astronomy (MPIA), the Max-Planck-Institute for Astrophysics (MPA), New Mexico State University, Ohio State University, University of Pittsburgh, University of Portsmouth, Princeton University, the United States Naval Observatory, and the University of Washington. This work is based in part on observations made at the following telescopes. The Hobby-Eberly Telescope (HET) is a joint project of the University of Texas at Austin, the Pennsylvania State University, Stanford University, Ludwig-Maximillians-Universität München, and GeorgAugust-Universität Göttingen. The HET is named in honor of its principal benefactors, William P. Hobby and Robert E. Eberly. The Marcario Low-Resolution Spectrograph is named for Mike Marcario of High Lonesome Optics, who fabricated several optical elements for the instrument but died before its completion; it is a joint project of the Hobby-Eberly Telescope partnership and the Instituto de Astronomía de la Universidad Nacional Autónoma de México. The Apache Point Observatory $3.5 \mathrm{~m}$ telescope is owned and operated by the Astrophysical Research Consortium. We thank the observatory director, Suzanne Hawley, and site manager, Bruce Gillespie, for their support of this project. The Subaru Telescope is operated by the National Astronomical Observatory of Japan. The William Herschel Telescope is operated by the Isaac Newton Group, on the island of La Palma in the Spanish Observatorio del Roque de los Muchachos of the Instituto de Astrofisica de Canarias. Based on observations made with ESO Telescopes at the La Silla or Paranal Observatories under programme IDs 77.A-0437, 78.A0325, 79.A-0715, and 80.A-0024. Based on observations made with the Nordic Optical Telescope, operated on the island of La Palma jointly by Denmark, Finland, Iceland, Norway, and Sweden, in the Spanish Observatorio del Roque de los Muchachos of the Instituto de Astrofisica de Canarias. Kitt Peak National Observatory, National Optical Astronomy Observatory, is operated by the Association of Universities for Research in Astronomy, Inc. (AURA) under cooperative agreement with the National Science Foundation. Based partially on observations made with the Italian Telescopio Nazionale Galileo (TNG) operated on the island of La Palma by the Fundación Galileo Galilei of the INAF (Istituto Nazionale di Astrofisica) at the Spanish Observatorio del Roque de los Muchachos of the Instituto de Astrofísica de Canarias. This work was supported in part by the Kavli Institute for Cosmological Physics at the University of Chicago through grants NSF PHY-0114422 and NSF PHY-0551142 and an endowment from the Kavli Foundation and its founder Fred Kavli. This work was also partially supported by the US Department of Energy through grant DEFG02-08ER41562 to Rutgers University (PI: S.W.J.). We thank Chris Miller for making the extended version of the $\mathrm{C} 4$ cluster catalog available. We thank Ben Koester for providing a catalog of cluster member galaxies for the maxBCG clusters. B.D. thanks B. Koester and S. Hansen for many invaluable discussions. 


\section{REFERENCES}

Abazajian, K., et al. 2004, AJ, 128, 502

Adelman-McCarthy, J. K., et al. 2007, ApJS, 172, 634

Alard, C., \& Lupton, R. H. 1998, ApJ, 503, 325

Aldering, G., et al. 2006, ApJ, 650, 510

Barbon, R. 1978, AJ, 83, 13

Blanton, M. R., \& Roweis, S. 2007, AJ, 133, 734

Blanton, M. R., et al. 2003a, AJ, 125, 2348

Blanton, M. R., et al. 2003b, ApJ, 592, 819

Cappellaro, E., Evans, R., \& Turatto, M. 1999, A\&A, 351, 459

Crane, P., Tammann, G. A., \& Woltjer, L. 1977, Nature, 265, 124

Della Valle, M., Panagia, N., Padovani, P., Cappellaro, E., Mannucci, F., \& Turatto, M. 2005, ApJ, 629, 750

Dilday, B., et al. 2008, ApJ, 682, 262

Dilday, B., et al. 2010, ApJ, 713, 1026

Forster, F., \& Schawinski, K. 2008, MNRAS, 388, L74

Frieman, J. A., et al. 2008, AJ, 135, 338

Fukugita, M., Ichikawa, T., Gunn, J. E., Doi, M., Shimasaku, K., \& Schneider, D. P. 1996, AJ, 111, 1748

Gal-Yam, A., Maoz, D., Guhathakurta, P., \& Filippenko, A. V. 2003, AJ, 125, 1087

Gal-Yam, A., Maoz, D., Guhathakurta, P., \& Filippenko, A. V. 2008, ApJ, 680, 550

Gal-Yam, A., Maoz, D., \& Sharon, K. 2002, MNRAS, 332, 37

Graham, M. L., et al. 2008, AJ, 135, 1343

Graham, M. L., et al. 2010, AJ, 139, 594

Greggio, L. 2005, A\&A, 441, 1055

Gunn, J. E., et al. 1998, AJ, 116, 3040

Gunn, J. E., et al. 2006, AJ, 131, 2332

Hansen, S. M., Sheldon, E. S., Wechsler, R. H., \& Koester, B. P. 2009, ApJ, 699,1333

Hogg, D. W., Finkbeiner, D. P., Schlegel, D. J., \& Gunn, J. E. 2001, AJ, 122, 2129

Holtzman, J. A., et al. 2008, AJ, 136, 2306

Ivezić, Ž., et al. 2004, Astron. Nachr., 325, 583
Jha, S., Riess, A. G., \& Kirshner, R. P. 2007, ApJ, 659, 122

Johnston, D. E., et al. 2007, arXiv:0709.1159

Jorgensen, I. 1997, MNRAS, 288, 161

Kaviraj, S., et al. 2007, ApJS, 173, 619

Koester, B. P., et al. 2007a, ApJ, 660, 239

Koester, B. P., et al. 2007b, ApJ, 660, 221

Lupton, R. H., Gunn, J. E., \& Szalay, A. S. 1999, AJ, 118, 1406

Mannucci, F. 2009, in AIP Conf. Ser. 1111, Probing Stellar Populations Out to the Distant Universe, ed. G. Giobbi et al. (Melville, NY: AIP), 467

Mannucci, F., Maoz, D., Sharon, K., Botticella, M. T., Della Valle, M., Gal-Yam, A., \& Panagia, N. 2008, MNRAS, 383, 1121

Menanteau, F., et al. 2010, arXiv:1002.2226

Miller, C. J., et al. 2005, AJ, 130, 968

Oyaizu, H., Lima, M., Cunha, C. E., Lin, H., \& Frieman, J. 2008, ApJ, 689 709

Padmanabhan, N., et al. 2004, New Astron., 9, 329

Prieto, J. L., et al. 2007, AJ, submitted (arXiv:0706.4088)

Sako, M., et al. 2008, AJ, 135, 348

Sand, D. J., Zaritsky, D., Herbert-Fort, S., Sivanandam, S., \& Clowe, D. 2008, AJ, 135, 1917

Schechter, P. L., Mateo, M., \& Saha, A. 1993, PASP, 105, 1342

Sharon, K., Gal-Yam, A., Maoz, D., Filippenko, A. V., \& Guhathakurta, P. 2007a, ApJ, 660, 1165

Sharon, K., et al. 2007b, in AIP Conf. Ser. 924, The Multicolored Landscape of Compact Objects and Their Explosive Origins, ed. T. di Salvo et al. (Melville, NY: AIP), 460

Sheldon, E. S., et al. 2009a, ApJ, 703, 2232

Sheldon, E. S., et al. 2009b, ApJ, 703, 2217

Smith, J. A., et al. 2002, AJ, 123, 2121

Stoughton, C., et al. 2002, AJ, 123, 485

Strateva, I., et al. 2001, AJ, 122, 1861

Strauss, M. A., et al. 2002, AJ, 124, 1810

Sullivan, M., et al. 2006, ApJ, 648, 868

Tucker, D. L., et al. 2006, Astron. Nachr., 327, 821

York, D. G., et al. 2000, AJ, 120, 1579

Zheng, C., et al. 2008, AJ, 135, 1766 